Pacific Journal of Mathematic 


\section{THE FREDHOLM EIGEN VALUES OF PLANE DOMAINS}

\section{SCHIFFER}

Introduction. The method of linear integral equations is an important tool in the theory of conformal mapping of plane simply-connected domains and in the boundary value problems of two-dimensional potential theory, in general. It yields a simple existence proof for solutions of such boundary value problems and leads to an effective construction of the required solution in terms of geometrically convergent NeumannLiouville series. The convergence quality of these series is of considerable practical importance and has been discussed by various authors $[4,5,6,7]$. It depends on the numerical value of the lowest nontrivial eigen value of the corresponding homogeneous integral equation which is an important functional of the boundary curve of the domain in question. Ahlfors $[1,10]$ gave an interesting estimate for this eigen value in terms of the extreme quasi-conformal mapping of the interior of this curve onto its exterior. Warschawski [15] gave a very useful estimate for it in terms of the corresponding eigen value of a nearby curve which allows often a good estimate of the desired eigen value in terms of a well-known one. This method is particularly valuable for special domains, for example, nearly-circular or convex ones.

It is the aim of the present paper to study the eigen functions and eigen values of the homogeneous Fredholm equation which is connected with the boundary value problem of two-dimensional potential theory. In particular, we want to obtain a sharp estimate for the lowest nontrivial eigen value in terms of function theoretic quantities connected with the curve considered. The steps of our investigation might become easier to understand by the following brief outline of our paper.

In $\S 1$ we define the eigen values and eigen functions considered and transform the basic integral equation into a form which exhibits more clearly the interrelation with analytic function theory and extend the eigen functions as harmonic functions into the interior and the exterior of the curve. The boundary relations between these harmonic extensions are discussed and utilized to provide an example of a set of eigen functions and eigen values for the case of ellipses.

In $\S 2$ we show the significance of the eigen value problem for the theory of the dielectric Green's function which depends on a positive parameter $\varepsilon$ and is defined in the interior as well as the exterior of the curve. This Green's function has an immediate electrostatic interpretation and its theoretical value consists in the fact that it permits a

Received June 29, 1956. Prepared under contract Nonr-225 (11), (NR-041-086) for Office of Naval Research. 
continuous transition from the Green's function of a domain to its Neumann's function. All dielectric Green's functions permit simple series developments in terms of the eigen functions and eigen values studied and the possible applications of these series developments to inequalities in potential theory are briefly indicated. Finally, it is shown that analytic completion of the dielectric Green's functions leads ultimately to univalent analytic functions in the interior as well as the exterior domain. This will lead, on the one hand, to interesting information on potential theoretical questions by use of the numerous distortion theorems of conformal mapping. On the other hand, we obtain in this way a one-parameter family of conformal maps of the domains which start with the identity and end up with the normalized mapping onto a circle. This parametrization is of importance in the theory of univalent functions; it is entirely different from the Löwner parametrization of univalent functions [8].

In $\S \S 3$ and 4 we derive formulas for the variation of the various eigen values and dielectric Green's functions. We use at first interior variations and are thus able to derive precise variational formulas with uniform estimates for the error terms. By superposition of interior variations and simple transformations we can easily derive variational formulas of the Hadamard type. It is seen that the variational formula for the dielectric Green's function is surprisingly similar to that for the ordinary Green's function. It is seen that the circle is a curve for which all nontrivial eigen values are infinite. Thus, the circle leads to a homogeneous integral equation with an eigen value of infinite degeneracy and the usual perturbation theory cannot be applied. We show, therefore, by a special argument how eigen values for nearlycircular domains can be obtained.

Finally, we apply in $\S 5$ the variational formula for the eigen values to a simple extremum problem for the lowest one which leads ultimately to the desired inequality. A characteristic difficulty, however, has to be overcome in this problem. It appears that the eigen values are only continuous functionals of the curve if the curve is deformed in such a way that normals in corresponding points are turned very little. Such a side condition is hard to preserve under general variations. We introduce, therefore, the concept of uniformly analytic curves which is closely related to the theory of univalent functions. Extremum problems within the class of uniformly analytic curves are easy to handle and the problem of the existence of extremum curves is likewise of very elementary nature. As the end result of our study an inequality then appears which estimates the lowest nontrivial Fredholm eigen value from below in terms of the modulus of uniform analyticity. This quantity is, however, easy to determine if a specific analytic curve 
is prescribed. It seems that the concept of uniform analyticity may play a useful role in many further extremum problems and variational investigations. As a side result of our study we obtain a new class of plane curves for which the Fredholm eigen functions and eigen values can be computed explicitly.

1. The Fredholm eigen values. Let $C$ be a closed curve in the complex $z$-plane which is three times continuously differentiable; we denote the interior of $C$ by $D$ and its exterior by $\tilde{D}$. The kernel

$$
k(z, \zeta)=\frac{\partial}{\partial n_{\zeta}} \log \frac{1}{|z-\zeta|}
$$

is a continuous function of both argument points as these vary on the curve $C$ only. We understand by $\frac{\partial}{\partial n_{\zeta}}$ the differentiation in direction of the normal at $\zeta$ on $C$ pointing into $D$.

The first boundary value problem of potential theory with respect to the domain $D$ can be reduced to the inhomogeneous integral equation

$$
f(z)=\phi(z)+\frac{1}{\pi} \int_{C} k(z, \zeta) \phi(\zeta) d s_{\zeta}, \quad z \in C,
$$

while the second boundary value problem can be solved by reduction to an integral equation with transposed kernel

$$
f(z)=\psi(z)-\frac{1}{\pi} \int_{C} k(\zeta, z) \psi(\zeta) d s_{\zeta}, \quad z \in C .
$$

In view of the Fredholm alternative in the theory of integral equations one is then led naturally to discuss the eigen values and eigen functions of the corresponding homogeneous integral equation

$$
\phi_{\nu}(z)=\frac{\lambda_{\nu}}{\pi} \int_{C} k(z, \zeta) \phi_{\nu}(\zeta) d s_{\zeta} .
$$

These functionals of $C$ play an important role in the potential theory of the domains $D$ and $\tilde{D}$ as shall be seen in the following considerations. In this section we shall give a brief survey of their theory and various transformations of the integral equation (3) which will be used later.

We introduce the harmonic function

$$
h_{\nu}(z)=\frac{\lambda_{\nu}}{\pi} \int_{C} k(z, \zeta) \phi_{\nu}(\zeta) d s_{\zeta}
$$


which is defined in $D$ and $\tilde{D}$; for the sake of clarity, we shall denote it by $\tilde{h}(z)$ if its argument point lies in $\tilde{D}$. By the well-known discontinuity behavior of the kernel (1), we have the limit relations, valid for an arbitrary point $z_{0} \in C$ :

$$
\lim _{z \rightarrow z_{0}} h_{\nu}(z)=\left(1+\lambda_{\nu}\right) \phi_{\nu}\left(z_{0}\right), \quad \lim _{z \rightarrow z_{0}} \tilde{h}_{\nu}(z)=\left(1-\lambda_{\nu}\right) \phi_{\nu}\left(z_{0}\right) .
$$

On the other hand, the normal derivative of a double-layer potential goes continuously through the curve $C$ which carries the charge and, hence,

$$
\frac{\partial}{\partial n} h_{\nu}(z)=-\frac{\partial}{\partial \tilde{n}} \tilde{h}_{\nu}(z), \quad \text { for } z \in C,
$$

where $\frac{\partial}{\partial \tilde{n}}$ denotes normal differentiation into $\tilde{D}$.

By Green's identity, we have

$$
\frac{1}{2 \pi} \int_{\sigma}\left[h_{\nu}(\zeta) \frac{\partial}{\partial n_{\zeta}} \log \frac{1}{|\zeta-z|}-\log \frac{1}{|\zeta-z|} \frac{\partial}{\partial n} h_{\nu}(\zeta)\right] d s_{\zeta}=h_{\nu}(z) \delta(z)
$$

and

$$
\left.\frac{1}{2 \pi} \int_{C} \tilde{h}_{\nu}(\zeta) \frac{\partial}{\partial \tilde{n}_{\zeta}} \log \frac{1}{|\zeta-z|}-\log \frac{1}{|\zeta-z|} \frac{\partial}{\partial \tilde{n}} \tilde{h}_{\nu}(\zeta)\right] d s_{\zeta}=\tilde{h}_{\nu}(z) \tilde{\delta}(z)
$$

where $\delta(z)$ and $\tilde{\delta}(z)$ are the characteristic functions of $D$ and $\tilde{D}$, that is,

$$
\delta(z)=\left\{\begin{array}{l}
1 \text { if } z \in D \\
0 \text { if } z \in \tilde{D}
\end{array}, \quad \tilde{\delta}(z)=1-\delta(z) .\right.
$$

Combining (7) with $\left(7^{\prime}\right)$ and observing the boundary relations (5) and (6) between $h_{\nu}(z)$ and $\tilde{h}_{\nu}(z)$, we obtain

$$
\begin{array}{ll}
-\frac{\lambda_{\nu}}{\pi\left(\lambda_{\nu}-1\right)} \int_{C} \log \frac{1}{|\zeta-z|} \frac{\partial}{\partial n} h_{\nu}(\zeta) d s_{\zeta}=h_{\nu}(z), & z \in D \\
-\frac{\lambda_{\nu}}{\pi\left(\lambda_{\nu}+1\right)} \int_{C} \log \frac{1}{|\zeta-z|} \frac{\partial}{\partial \tilde{n}} \tilde{h}_{\nu}(\zeta) d s_{\zeta}=\tilde{h}_{\nu}(z), & z \in \tilde{D} .
\end{array}
$$

Define two analytic functions in $D$ and $\tilde{D}$, respectively, by the formulas

$$
v_{\nu}(z)=\frac{\partial}{\partial z} h_{\nu}(z), \quad \tilde{v}_{\nu}(z)=\frac{\partial}{\partial z} \tilde{h}_{\nu}(z)
$$

Differentiating (9) and (10) with respect to $z$, one obtains easily 


$$
v_{\nu}(z)=\frac{\lambda_{\nu}}{2 \pi i} \int_{\sigma} \frac{\overline{\left(v_{\nu}(\zeta) d \zeta\right)}}{\zeta-z}, \tilde{v}_{\nu}(z)=\frac{\lambda_{\nu}}{2 \pi i} \int_{C} \frac{\overline{\left(\tilde{v}_{\nu}(\zeta) d \zeta\right)}}{\zeta-z}
$$

These are elegant integral equations for the analytic functions $v_{\nu}(z)$ and $\tilde{v}_{\nu}(z)$ which are valid for $z \in D$ and $z \in \tilde{D}$, respectively.

We can also bring (12) into the form

$$
v_{\nu}(z)=\frac{\lambda_{\nu}}{\pi} \iint_{D} \frac{\overline{v_{\nu}(\zeta)}}{(\zeta-z)^{2}} d \tau \quad \text { for } z \in D
$$

and

$$
\tilde{v}_{\nu}(z)=\frac{\lambda_{\nu}}{\pi} \iint_{\tilde{D}} \frac{\overline{\tilde{v}_{\nu}(\zeta)}}{(\zeta-z)^{2}} d \tau
$$

for $z \in \tilde{D}$

which expresses $v_{\nu}(z)$ and $\tilde{v}_{\nu}(z)$ as solutions of integral equations with improper kernels. The integrals involved have to be understood in the Cauchy principal value sense.

In the transition from the integral equations (3) defined on $C$ to the integral equations (13), $\left(13^{\prime}\right)$ defined in $D$ and $\tilde{D}$, we have lost one particular eigen function. Indeed, if $h(z)=$ const. were one of our eigen functions $h_{\nu}(z)$ it would have been cancelled out in the differentiation (11). But by (5), $h_{\nu}(z)=$ const. implies $\phi_{\nu}(z)=$ const. on $C$ and from (3) and the identity

$$
\frac{1}{\pi} \int_{C} k(z, \zeta) d s_{\zeta}=1, \quad z \in C,
$$

follows, in fact, that each constant is an eigen function of (3) with the eigen value $\lambda=+1$. The eigen value $\lambda=+1$ plays an exceptional role in the entire Poincaré-Fredholm theory of the boundary value problem; the fact that the equations (13), $\left(13^{\prime}\right)$ lead to all other eigen values and their corresponding eigen functions and eliminate $\lambda=+1$ represents, therefore, a strong argument in favor of this transition.

Let $z(s)$ be the parametric representation of $C$ in terms of its length parameter $s$. Then

$$
z^{\prime}=\frac{d z}{d s}
$$

will be the unit vector in tangential direction to $C$. The boundary relations (5) and (6) for $h_{\nu}$ and $\tilde{h}_{\nu}$ go over into the equations on $C$ :

$$
\Re\left\{v_{\nu}(z) z^{\prime}\right\}=\frac{1+\lambda_{\nu}}{1-\lambda_{\nu}} \Re\left\{\tilde{v}_{\nu}(z) z^{\prime}\right\}
$$




$$
\mathfrak{\Im}\left\{v_{\nu}(z) z^{\prime}\right\}=\mathfrak{S}\left\{\tilde{v}_{\nu}(z) z^{\prime}\right\}
$$

which can be combined into the one complex equation

$$
v_{\nu}(z) z^{\prime}=\frac{1}{1-\lambda_{\nu}} \tilde{v}_{\nu}(z) z^{\prime}+\frac{\lambda_{\nu}}{1-\lambda_{\nu}} \overline{\tilde{v}}_{\nu}(z) z^{\prime}
$$

This relation combined with (12) throws an interesting light on the connection between $v_{\nu}(z)$ and $\tilde{v}_{\nu}(z)$. In fact, if we insert (18) into the first equation (12) and apply Cauchy's theorem, we find

$$
v_{\nu}(z)=\frac{1}{2 \pi i} \frac{\lambda_{\nu}}{1-\lambda_{2}} \int_{C} \frac{\overline{\left(\tilde{v}_{\nu}(\zeta) d \zeta\right)}}{\zeta-z}, \quad z \in D
$$

Observe that the second formula (12) yields $\tilde{v}_{\nu}(z)$ for $z \in \tilde{D}$; now we see that the same expression yields $v_{\nu}(z)$ for $z \in D$, except for the factor $1-\lambda_{\nu}$. Similarly, one shows easily

$$
\tilde{v}_{\nu}(z)=\frac{1}{2 \pi i} \frac{\lambda_{\nu}}{1+\lambda_{\nu}} \int_{C} \frac{\left.\overline{\left(v_{\nu}(\zeta) d \zeta\right.}\right)}{\zeta-z}, \quad z \in \tilde{D}
$$

If $f(z)$ is an arbitrary complex-valued function in the entire $z$-plane of the class $\mathscr{L}^{2}$, the equation

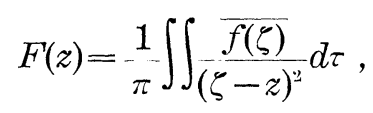

defines a new function in $\mathscr{L}^{2}$, its Hilbert transform. It is well-known [2] that the Hilbert transformation is norm-preserving, that is,

$$
\iint|F|^{2} d \tau=\iint|f|^{2} d \tau
$$

Our formulas (13), (13') and (19), (20) imply that the functions

$$
f_{\nu}(z)=\left\{\begin{array}{cc}
v_{\nu}(z) \text { in } D \\
0 \text { in } \tilde{D}
\end{array} \text { and } \tilde{f}_{\nu}(z)=\left\{\begin{array}{cc}
0 & \text { in } D \\
\tilde{v}_{\nu}(z) & \text { in } \tilde{D}
\end{array}\right.\right.
$$

have the Hilbert transforms

$$
F_{\nu}(z)=\left\{\begin{array}{ll}
\frac{1}{\lambda_{\nu}} v_{\nu}(z) & \text { in } D \\
\left(\frac{1}{\lambda_{\nu}}+1\right) \tilde{v}_{\nu}(z) & \text { in } \tilde{D}
\end{array} \text { and } \quad \tilde{F}_{\nu}(z)= \begin{cases}\left(\frac{1}{\lambda_{\nu}}-1\right) v_{\nu}(z) & \text { in } D \\
\frac{1}{\lambda_{\nu}} \tilde{v}_{\nu}(z) & \text { in } \tilde{D} .\end{cases}\right.
$$

Hence (22) yields 


$$
\left(\lambda_{\nu}-1\right) \iint_{D}\left|v_{\nu}\right|^{2} d \tau=\left(\lambda_{\nu}+1\right) \iint_{\tilde{D}}\left|\widetilde{v}_{\nu}\right|^{2} d \tau
$$

From (25) we conclude easily that

$$
\left|\lambda_{2}\right|>1
$$

For if, for example, $\lambda_{\nu}=1$, we would have $\tilde{v}_{\nu} \equiv 0$ in $\tilde{D}, \tilde{h_{\nu}}(z)=$ const. and hence by (6) also $h_{\nu}(z)=$ const. But this would imply, in turn, $v_{\nu}(z) \equiv 0$ and no eigen functions would exist.

With each eigen value $\lambda_{\nu}$ of (4) the eigen value $-\lambda_{\nu}$ also occurs, except for $\lambda=1$. In fact, if we denote the conjugate functions of $h_{\nu}(z)$ and $\tilde{h}_{\nu}(z)$ by $g_{\nu}(z)$ and $\tilde{g}_{\nu}(z)$, we have by the Cauchy-Riemann formulas the relations

$$
\frac{\partial}{\partial n} g_{\nu}(z)=-\frac{1+\lambda_{\nu}}{1-\lambda_{\nu}} \frac{\partial}{\partial \tilde{n}} \tilde{g}_{\nu}(z), \frac{\partial}{\partial s} g_{\nu}(z)=\stackrel{\partial}{\partial s} \tilde{g}_{\nu}(z)
$$

Hence, putting

$$
g_{\nu}^{*}(z)=\left(1-\lambda_{\nu}\right) g_{\nu}(z), \quad \tilde{g}_{\nu}^{*}(z)=\left(1+\lambda_{\nu}\right) \tilde{g}_{\nu}(z)
$$

and adding an appropriate constant we find for $z \in C$ :

$$
g_{\nu}^{*}(z)=\frac{1-\lambda_{\nu}}{1+\lambda_{\nu}} \tilde{g}_{\nu}^{*}(z), \quad \frac{\partial}{\partial n} g_{\nu}^{*}(z)=-\frac{\partial}{\partial \tilde{n}} \tilde{g}_{\nu}^{*}(z) .
$$

These are the boundary relations between $h_{\nu}$ and $\tilde{h}_{\nu}$ but with $-\lambda_{\nu}$ instead of $\lambda_{\nu}$. This proves our assertion.

If we start conversely from the complex integral equations (13) and $\left(13^{\prime}\right)$ and consider any eigen function $v_{\nu}(z)$ with the eigen value $\lambda_{\nu}$, it will be observed that $e^{i \alpha} v_{\nu}(z)$ is also an eigen function to the eigen value $\lambda_{2} e^{-2 i \alpha}$. Hence, if we focus our attention on the integral equations (12) or (13) we may assume without loss of generality that $\lambda_{\nu}$ is a real positive eigen value. Calculating backward, we can easily see that each such eigen value is also an eigen value of the Fredholm integral equation (4) and so is $-\lambda_{\nu}$.

It is readily verified that eigen functions $v_{\nu}(z)$ and $v_{\mu}(z)$ which belong to different eigen values $\lambda_{\nu}$ and $\lambda_{\mu}$ satisfy the orthogonality relation

$$
\iint_{D} v_{\nu}(z) \overline{v_{\mu}(z)} d \tau=0 .
$$

This condition can be extended to the case of any two linearly independent eigen functions. Similarly

$$
\iint_{\tilde{D}} \tilde{v}_{\nu} \overline{\tilde{v}}_{\mu} d \tau=0
$$


for any two different eigen functions $\tilde{v}_{\nu}, \tilde{v}_{\mu}$. In view of (25), we define

$$
w_{\nu}(z)=\sqrt{\lambda_{\nu}-1} v_{\nu}(z), \quad \tilde{w}_{\nu}(z)=i \sqrt{\lambda_{\nu}+1} \tilde{v}_{\nu}(z) .
$$

Then we can assume the orthonormality relations

$$
\iint_{D} w_{\nu} \bar{w}_{\mu} d \tau=\delta_{\nu \mu}, \iint_{\tilde{D}} \tilde{w}_{\nu} \overline{\tilde{w}}_{\mu} d \tau=\delta_{\nu \mu} .
$$

We have in view of (18) the boundary relations on $C$ :

$$
w_{\nu}(z) z^{\prime}=\frac{i}{\sqrt{\lambda_{\nu}^{2}-1}} \tilde{w}_{\nu}(z) z^{\prime}-\frac{\lambda_{\nu} i}{\sqrt{\lambda_{\nu}^{2}-1}} \overline{\tilde{w}}_{\nu}(z) z^{\prime} ;
$$

from (19) and (20) follows

$$
w_{\nu}(z)=-\frac{\lambda_{\nu}}{2 \pi \sqrt{\lambda_{\nu}^{2}-1}} \int_{C} \frac{\overline{\left(\tilde{w}_{\nu}(\zeta) d \zeta\right)}}{\zeta-z},
$$

and

$$
\tilde{w}_{\nu}(z)=\frac{\lambda_{\nu}}{2 \pi \sqrt{\lambda_{\nu}^{2}-1}} \int_{c} \frac{\overline{\left(w_{\nu}(\zeta) d \zeta\right)}}{\zeta-z}, \quad z \in \tilde{D}
$$

If we were able to guess two functions $w(z)$ and $\tilde{w}(z)$ which are analytic in $D$ and $\tilde{D}$, respectively, and which satisfy on $C$ the relation (34) for a properly chosen $\lambda$, we would have obtained a particular solution for the eigen value problems (13) and $\left(13^{\prime}\right)$. It is sometimes possible to construct such pairs of functions and to obtain thus eigen values and eigen functions for the Fredholm integral equation. One possibility of construction is the following: We refer the curve $C$ by conformal mapping to the unit circumference. Let

$$
z=f(\zeta)
$$

be analytic on and near $|\zeta|=1$ and map it onto $C$. The condition (34) can now be referred to $|\zeta|=1$ and reads:

$$
w[f(\zeta)] f^{\prime}(\zeta) i \zeta=-\frac{1}{\sqrt{\lambda^{2}-1}} \tilde{w}[f(\zeta)] f^{\prime}(\zeta) \zeta-\frac{\lambda}{\sqrt{\lambda^{2}-1}} \tilde{\tilde{w}}[f(\zeta)] f^{\prime}(\zeta) \zeta .
$$

Since the conjugation $\bar{\zeta}$ means just $\frac{1}{\zeta}$ on $|\zeta|=1$ it is easier to guess solutions in this form.

Let, for example,

$$
z=f(\zeta)=\zeta+\frac{\rho^{2}}{\zeta}, \quad 0<\rho<1
$$


This means that $C$ is an ellipse. Let us put

$$
w[f(\zeta)] f^{\prime}(\zeta) \zeta=a \zeta^{n}+b \zeta^{-n}
$$

and

$$
w[f(\zeta)] f^{\prime}(\zeta) \zeta=i \zeta^{-n} .
$$

Condition (38) will be fulfilled if we put

$$
a=\frac{\lambda}{\sqrt{\lambda^{2}-1}}, \quad b=\frac{-1}{\sqrt{\lambda^{2}}-1} .
$$

Define $W(f)=\int w d f$ and $\tilde{W}(f)=\int \tilde{w} d f$. Then (40) and (41) yield

$$
\begin{aligned}
& W[f(\zeta)]=\frac{1}{n}\left(a \zeta^{n}-b \zeta^{-n}\right)=\frac{\lambda}{n \sqrt{\lambda^{2}-1}}\left(\zeta^{n}+\lambda^{-1} \zeta^{-n}\right), \\
& \tilde{W}[f(\zeta)]=-\frac{i}{n} \zeta^{-n} .
\end{aligned}
$$

Now the function (39) is univalent outside of $|\zeta|=\rho$ and, hence, we may consider $\zeta$ as a regular analytic function of $z \in \tilde{D}$. Thus, $\tilde{W}(z)$ and $\tilde{w}(z)$ are regular analytic in $\tilde{D}$. In order that $W(z), w(z)$ be analytic in $D$ we must require that

$$
\lambda=\rho^{-2 n} \text {. }
$$

In fact, $\zeta^{n}+\frac{\rho^{2 n}}{\zeta^{n}}$ can be expressed as a Chebysheff polynomial of $z$. Thus we have guessed an infinity of eigen values and eigen functions for the case of the ellipse. It can be shown that $\lambda^{n}= \pm \rho^{-2 n}$ gives all eigen values of the ellipse for $n=1,2, \cdots$. Since $\rho=0$ describes a circle, we recognize, in particular, that all eigen values $\lambda_{2}$ for a circle have the the value infinity [3].

If we know the eigen values and eigen functions of a given domain $D$ we can find immediately the eigen values and eigen functions of every domain $D^{*}$ which is obtained from $D$ by a linear transformation

$$
z^{*}=\frac{a z+b}{c z+d}=l(z)
$$

In fact, let

$$
w_{\nu}^{*}\left(z^{*}\right) l^{\prime}(z)=w_{\nu}(z), \quad \tilde{w}_{\nu}^{*}\left(z^{*}\right) l^{\prime}(z)=\tilde{w}_{\nu}(z), \quad \lambda_{\nu}^{*}=\lambda_{\nu} .
$$


It follows from (33) that the $w_{\nu}^{*}$ and $\tilde{w}_{\nu}^{*}$ form an orthonormal set of analytic functions in $D^{*}$ and $\tilde{D}^{*}$, respectively. Since we have on $C^{*}$

$$
z^{* \prime}(z)=l^{\prime}(z)
$$

it is also obvious that (34) is fulfilled which shows that $w_{\nu}^{*}$ and $\tilde{w}_{\nu}^{*}$ are, in fact, the normalized eigen functions of the domains $D^{*}$ and $\tilde{D}^{*}$. In particular, we note that the eigen values $\lambda_{\nu}$ of a domain are unchanged under linear transformation. Similar domains, for example, have the same set of eigen values.

2. The dielectric problem. The consideration of the electrostatic field of a point source at $\zeta$ in the presence of a dielectric medium in $D$ with the dielectric constant $\varepsilon$ leads to the following heuristic definition of a Green's function $G_{\varepsilon}(z, \zeta)$ :

(a) $G_{\varepsilon}(z, \zeta)$ is harmonic in $D$ and $\tilde{D}$, except for $z=\zeta$.

(b) $G_{\varepsilon}(z, \zeta)-\log \frac{1}{|z-\zeta|}$ is harmonic at $\zeta$ if $\zeta \in \tilde{D}$.

(b') $G_{\varepsilon}(z, \zeta)-\varepsilon \log \frac{1}{|z-\zeta|}$ is harmonic at $\zeta$ if $\zeta \in D$.

(c) $G_{\varepsilon}(z, \zeta)$ is continuous through $C$.

(d) $\frac{\partial}{\partial n} G_{\varepsilon}(z, \zeta)+\varepsilon \frac{\partial}{\partial \tilde{n}} G_{\varepsilon}(z, \zeta)=0$ on $C$ for $\zeta \in D$ or $\tilde{D}$.

(e) $\log |z|+G_{\varepsilon}(z, \zeta) \rightarrow 0$ if $z \rightarrow \infty$, for $\zeta \in D$ or $\zeta \in \tilde{D}$.

It is easily seen that $G_{\varepsilon}(z, \zeta)$ is uniquely determined by these conditions and that it satisfies the symmetry condition

$$
G_{\varepsilon}(\zeta, \eta)=G_{\varepsilon}(\eta, \zeta)
$$

We may construct $G_{\varepsilon}(z, \zeta)$ by means of a line potential as follows. Let $\zeta \in \tilde{D}$ and put

$$
G_{\varepsilon}(z, \zeta)=\log \frac{1}{|z-\zeta|}+\int_{C} \mu(\eta, \zeta) \log |\eta-z| d s_{\eta} .
$$

This set-up satisfies automatically conditions (a), (b) and (c); we can fulfill condition (e) by the requirement

$$
\int_{c} \mu(\eta, \zeta) d s_{\eta}=0
$$

and finally $(d)$ by solving the integral equation

$$
\text { (4) }-\frac{1-\varepsilon}{1+\varepsilon} \cdot \frac{1}{\pi} \frac{\partial}{\partial n_{z}} \log \frac{1}{|z-\zeta|}=\mu(z, \zeta)-\frac{1-\varepsilon 1}{1+\varepsilon \pi} \int_{C} \mu(\eta, \zeta) \frac{\partial}{\partial n_{z}} \log \frac{1}{|\eta-z|} d s_{\eta} \text {. }
$$


As long as $\varepsilon>0$ this equation can be solved in a unique way since $\left|\frac{1-\varepsilon}{1+\varepsilon}\right|<1$ and all eigen values of the corresponding homogeneous integral equation are larger or equal to one in absolute value. One verifies also from (4) that condition (3) is automatically fulfilled. In a similar way we proceed for $\zeta \in D$.

The integral equation (4) indicates already the close relation between the Green's function $G_{\varepsilon}(z, \zeta)$ and the Fredholm eigen functions. We obtain a further insight from the Dirichlet identities:

$$
\begin{aligned}
\iint_{D} \nabla G_{\varepsilon}(z, \zeta) \nabla h_{\nu}(z) d \tau & =-\int_{C} G_{\varepsilon}(z, \zeta) \frac{\partial}{\partial n} h_{\nu}(z) d s_{z} \\
& =2 \pi \varepsilon h_{\nu}(\zeta) \delta(\zeta)-\int_{C} h_{\nu}(z) \frac{\partial}{\partial n} G_{\varepsilon}(z, \zeta) d s_{z}
\end{aligned}
$$

and

$$
\begin{aligned}
\iint_{\tilde{D}} \nabla G_{\varepsilon}(z, \zeta) \cdot \nabla \tilde{h}_{\nu}(z) d \tau & =-\int_{C} G_{\varepsilon}(z, \zeta) \frac{\partial}{\partial \tilde{n}} \tilde{h}_{\nu}(z) d s_{z} \\
& =2 \pi \tilde{h}_{\nu}(\zeta) \tilde{\delta}(\zeta)-\int_{C} \tilde{h}_{\nu}(z) \frac{\partial}{\partial \tilde{n}} G_{\varepsilon}(z, \zeta) d s_{z} .
\end{aligned}
$$

Here we use $\delta(\zeta)$ and $\tilde{\delta}(\zeta)$ as defined in (1.8). Identity (6) is valid in spite of the logarithmic pole of $G_{\varepsilon}$ at infinity since $\tilde{h}_{\nu}(z)$ vanishes there. Adding (5) and (6) and using (1.6), we obtain

$$
\iint_{\tilde{D}} \nabla G_{\varepsilon}(z, \zeta) \nabla \tilde{h}_{\nu}(z) d \tau=-\iint_{D} \nabla G_{\varepsilon}(z, \zeta) \nabla h_{\nu}(z) d \tau
$$

Putting

$$
\rho_{\nu}=\frac{\lambda_{\nu}+1}{\lambda_{\nu}-1}
$$

and using the boundary relations (1.5) and (d), we find:

$$
\begin{aligned}
-\varepsilon \rho_{\nu} \iint_{\tilde{D}} \nabla G_{\varepsilon}(z, \zeta) \nabla \tilde{h}_{\nu} d \tau & +\iint_{D} \nabla G_{\varepsilon}(z, \zeta) \nabla h_{\nu} d \tau \\
& =2 \pi \varepsilon\left[h_{\nu}(\zeta) \delta(\zeta)-\rho_{\nu} \tilde{h}_{\nu}(\zeta) \tilde{\delta}(\zeta)\right] .
\end{aligned}
$$

Thus, finally,

$$
\begin{aligned}
\iint_{D} \nabla G_{\varepsilon}(z, \zeta) \nabla h_{\nu}(z) d \tau & =\frac{2 \pi \varepsilon}{1+\varepsilon \rho_{\nu}}\left[h_{\nu}(\zeta) \delta(\zeta)-\rho_{\nu} \tilde{h}_{\nu}(\zeta) \tilde{\delta}(\zeta)\right] \\
& =-\iint_{\tilde{D}} \nabla G_{\varepsilon}(z, \zeta) \nabla \tilde{h}_{\nu} d \tau
\end{aligned}
$$


The eigen functions $h_{\nu}(z)$ connected with the Fredholm equation appear thus as the eigen functions of the integral equation in $D$ :

$$
\frac{1+\varepsilon \rho_{\nu}}{2 \pi \varepsilon} \iint_{D} \nabla G_{\varepsilon}(z, \zeta) \cdot \nabla h_{\nu}(z) d \tau=h_{\nu}(\zeta), \quad \zeta \in D
$$

Let $G(z, \zeta)$ be the ordinary Green's function of $D$; obviously

$$
\iint_{D} \nabla G(z, \zeta) \cdot \nabla h_{\nu}(z) d \tau=0
$$

Hence we obtain for $h_{\nu}(z)$ the integral equation

$$
\frac{1+\varepsilon \rho_{\nu}}{2 \pi \varepsilon} \iint_{D} \nabla K_{\varepsilon}(z, \zeta) \cdot \nabla h_{\nu}(z) d \tau=h_{\nu}(\zeta)
$$

with the regular harmonic kernel

$$
K_{\varepsilon}(z, \zeta)=G_{\varepsilon}(z, \zeta)-\varepsilon G(z, \zeta) .
$$

Let

$$
\tilde{G}(z, \infty)=\log |z|+\tilde{\gamma}+O\left(\frac{1}{|z|}\right)
$$

represent the Green's function of $\tilde{D}$ with the source point at infinity. By (1.5) we have obviously

$$
\int_{C} h_{\nu}(z) \frac{\partial \tilde{G}(z, \infty)}{\partial \tilde{n}} d s=\frac{1+\lambda_{\nu}}{1-\lambda_{\nu}} \int_{C} \tilde{h}_{\nu}(z) \frac{\partial \tilde{G}(z, \infty)}{\partial \tilde{n}}=2 \pi \frac{1+\lambda_{\nu}}{1-\lambda_{\nu}} \tilde{h}_{\nu}(\infty)=0 .
$$

We now define the linear space $\Sigma$ consisting of all functions $h(z)$ which are harmonic in $D$, have a finite Dirichlet integral there and satisfy the linear homogeneous condition

$$
\int_{C} h(z) \frac{\partial \widetilde{G}(z, \infty)}{\partial \tilde{n}} \frac{\infty}{d s=0 .}
$$

Observe that the only constant element in $\Sigma$ is the function $h \equiv 0$. All $h_{\nu}(z)$ lie in $\Sigma$; in view of $(12)$ and the symmetry of $K_{\varepsilon}(z, \zeta)$ we may assume that they are orthonormalized by the conditions

$$
\iint_{D} \nabla h_{\nu} \cdot \nabla h_{\mu} d \tau=\delta_{\nu \mu}
$$

and it is easily seen that they form a complete orthonormal set in $\Sigma$ [3].

If we use the conditions (c) and (e) in the definition of $G_{\varepsilon}(z, \zeta)$, we can show that the function 


$$
h(z)=K_{\varepsilon}(z, \zeta)-\tilde{\gamma}
$$

lies in $\Sigma$. Hence we have for it the following series development

$$
G_{\varepsilon}(z, \zeta)-\varepsilon G(z, \zeta)=\tilde{\gamma}+\sum_{\nu=1}^{\infty} \frac{h_{\nu}(z) h_{\nu}(\zeta)}{1+\varepsilon \rho_{\nu}} \cdot 2 \pi \varepsilon, \quad \zeta \in D .
$$

The Fourier coefficients in this development have been calculated from (12); the series converges uniformly in each closed subdomain of $D$.

Suppose next $\zeta \in \tilde{D}$ and consider the harmonic function

$$
h(z)=G_{\varepsilon}(z, \zeta)+\tilde{G}(\zeta, \infty)-\tilde{\gamma} .
$$

It is easily seen that $h(z) \in \Sigma$. Hence we may develop $h(z)$ into a series in the complete orthonormal system $h_{\nu}(z)$. Using (10), we find

$$
G_{\varepsilon}(z, \zeta)=\tilde{\gamma}-\tilde{G}(\zeta, \infty)-2 \pi \varepsilon \sum_{\nu=1}^{\infty} \frac{\rho_{\nu}}{1+\varepsilon \rho_{\nu}} h_{\nu}(z) \tilde{h}_{\nu}(\zeta) .
$$

This series converges for $\zeta \in \tilde{D}$ and $z$ in a closed subdomain of $D$.

Observe that by definition of $G_{\varepsilon}(z, \zeta)$ we have for $\varepsilon=1$

$$
G_{1}(z, \zeta)=\log \frac{1}{|z-\zeta|}
$$

Hence (19) contains the following series representation for the ordinary Green's function of $D$ :

$$
G(z, \zeta)=\log \frac{1}{|z-\zeta|}-\tilde{\gamma}-2 \pi \sum_{\nu=1}^{\infty} \frac{h_{\nu}(z) h_{\nu}(\zeta)}{1+\rho_{\nu}}
$$

On the other hand, (21) reduces for $\varepsilon=1$ to

$$
\tilde{G}(\zeta, \infty)=\log |z-\zeta|+\tilde{\gamma}-2 \pi \sum_{\nu=1}^{\infty} \frac{\rho_{\nu}}{1+\rho_{\nu}} h_{\nu}(z) \tilde{h}_{\nu}(\zeta) .
$$

In a similar way we can derive series developments for $G_{\varepsilon}(z, \zeta)$ in the exterior $\tilde{D}$ of $C$. Observe that in view of the boundary conditions (1.5) and (1.6) the normalization (17) of the $h_{\nu}(z)$ implies

$$
\iint_{\tilde{D}} \nabla \tilde{h}_{\nu} \cdot \nabla \tilde{h}_{\mu} d \tau=\rho_{\nu}^{-1} \delta_{\mu \nu} .
$$

Let $\tilde{\Sigma}$ be the linear function space consisting of all functions $\tilde{h}(z)$ which are harmonic in $\tilde{D}$, have a finite Dirichlet integral there and which vanish at infinity. Clearly the $\left\{\rho_{\nu}^{1 / 2} \tilde{h}_{\nu}(z)\right\}$ form a complete orthonormal set in $\tilde{\Sigma}$. 
On the other hand, let $\tilde{G}(z, \zeta)$ be the Green's function of $\tilde{D}$. Then it is easily verified that by condition (e) and (14)

$$
\tilde{h}(z)=G_{\varepsilon}(z, \zeta)-\tilde{G}(z, \zeta)+\tilde{G}(\zeta, \infty)+\tilde{G}(z, \infty)-\tilde{\gamma}, \quad z, \zeta \in \tilde{D},
$$

lies in $\tilde{\Sigma}$. Again using the Dirichlet formula (10), we find

$$
\begin{gathered}
G_{\varepsilon}(z, \zeta)-\tilde{G}(z, \zeta)+\tilde{G}(\zeta, \infty)+\tilde{G}(z, \infty)-\tilde{\gamma} \\
=2 \pi \varepsilon \sum_{\nu=1}^{\infty} \frac{\tilde{h}_{\nu}(z) \tilde{h}_{\nu}(\zeta) \underline{\rho_{\nu}^{2}}}{1+\varepsilon \rho_{\nu}} .
\end{gathered}
$$

Putting, in particular, $\varepsilon=1$, we obtain by virtue of (22):

$$
\tilde{G}(z, \zeta)-\tilde{G}(\zeta, \infty)-\tilde{G}(z, \infty)=\log \frac{1}{|z-\zeta|}-\tilde{\gamma}-2 \pi \sum_{\nu=1}^{\infty} \frac{\tilde{h}_{\nu}(z) \tilde{h}_{\nu}(\zeta) \rho_{\nu}}{1+\rho_{\nu}} .
$$

We have thus shown that all dielectric Green's functions can be constructed simultaneously and in $D$ as well as in $\tilde{D}$, once the system of eigen functions $h_{\nu}(z)$ and the corresponding eigen values $\lambda_{\nu}$ are known.

Numerous inequalities can be drawn from these representations. We shall restrict ourselves to one single example. Denote, for $\zeta \in D$,

$$
G_{\varepsilon}(z, \zeta)-\varepsilon \log \frac{1}{|z-\zeta|}=g_{\varepsilon}(z, \zeta), \quad G(z, \zeta)-\log \frac{1}{|z-\zeta|}=g(z, \zeta)
$$

The functions $g_{\varepsilon}(z, \zeta)$ and $g(z, \zeta)$ represent the potentials induced by a unit pole at $\zeta$ in the presence of the dielectric in $D$, and in the presence of the grounded conductor $C$, respectively. We find from (19)

$$
g_{\varepsilon}(\zeta, \zeta)-\varepsilon g(\zeta, \zeta) \geqq \tilde{\gamma} .
$$

Since $e^{-\tilde{\gamma}}$ represents the electrostatic capacity of the conductor $C$, we obtain an interesting estimate for the dielectric reaction potential in terms of capacity constants connected with the conductor surface $C$. For $\varepsilon=1$, we have $g_{1}(\zeta, \zeta)=0$ and hence

$$
\tilde{\gamma} \leqq-g(\zeta, \zeta) .
$$

This is an inequality connecting the inner and the outer Green's function of $C$; in the case that $C$ is a circumference and $\zeta$ is its center, this inequality becomes an equality.

Up to this point we stressed the connection between the Green's function $G(z, \zeta)$ and the eigen functions $h_{\nu}(z)$. Since the Fredholm eigen functions appear also in the theory of the second boundary value problem, we should also expect some relations between the $h_{\nu}(z)$ and the Neumann's function of the domain $D$. 
The Neumann's function is usually defined by its constant normal derivative on $C$

$$
\frac{\partial N(z, \zeta)}{\partial n_{z}}=\frac{2 \pi}{L}, \quad z \in C, \zeta \in D, L=\text { length of } C
$$

and by the linear homogeneous side condition

$$
\int_{0} N(z, \zeta) d s_{z}=0
$$

In order to operate within the class $\Sigma$, characterized by (16), we introduce the functions

$$
\alpha(z)=\frac{1}{2 \pi} \int_{C} N(t, z)\left[\frac{\partial \tilde{G}(t, \infty)}{\partial n}+\frac{2 \pi}{L}\right] d s_{t}
$$

and

$$
\begin{aligned}
h(z)= & N(z, \zeta)-G(z, \zeta)+\alpha(z)+\alpha(\zeta) \\
& +\frac{1}{4 \pi^{2}} \iint_{C C} N(t, \tau) \frac{\partial \tilde{G}(t, \infty)}{\partial n} \frac{\partial \tilde{G}(\tau, \infty)}{\partial n} d s_{t} d s_{\tau} .
\end{aligned}
$$

It is easily verified that $h(z) \in \Sigma$. Since obviously

$$
\int_{c}\left[\frac{\partial \tilde{G}(t, \infty)}{\partial n}+\frac{2 \pi}{L}\right] d s=0,
$$

the function $\alpha(z)$ is harmonic in $D$ and has the normal derivative

$$
\frac{\partial \alpha}{\partial n}=-\frac{\partial \tilde{G}(z, \infty)}{\partial n}-\frac{2 \pi}{L},
$$

Hence, finally, we have for $z \in C$

$$
\frac{\partial h}{\partial n}=-\frac{\partial G(z, \zeta)}{\partial n}-\frac{\partial \tilde{G}(z, \infty)}{\partial n}
$$

and consequently in view of (16) valid for each $h_{\nu}(z)$ :

$$
\iint_{D} \nabla h \cdot \nabla h_{\nu} d \tau=-\int_{C} h_{\nu} \frac{\partial h}{\partial n} d s=2 \pi h_{\nu}(\zeta) .
$$

Since $h(z) \in \Sigma$ and the $h_{\nu}(z)$ are a complete orthonormal system in $\Sigma$, we have the Fourier development 


$$
\begin{aligned}
N(z, \zeta) & -G(z, \zeta)+\frac{1}{2 \pi} \int_{C} N(t, z) \frac{\partial \tilde{G}(t, \infty)}{\partial n} d s+\frac{1}{2 \pi} \int_{C} N(t, \zeta) \frac{\partial \tilde{G}(t, \infty)}{\partial n} d s \\
& +\frac{1}{4 \pi^{2}} \iint_{C C} N(t, \tau) \frac{\partial \tilde{G}(t, \infty)}{\partial n} \frac{\partial \tilde{G}(\tau, \infty)}{\partial n} d s_{t} d s_{\tau}=\sum_{\nu=1}^{\infty} h_{\nu}(z) h_{\nu}(\zeta) \cdot 2 \pi
\end{aligned}
$$

This formula is useful to establish the exact asymptotics of the function $G_{\varepsilon}(z, \zeta)$ as $\varepsilon \rightarrow 0$ as can be seen from formula (19).

The dielectric Green's functions $G_{\varepsilon}(z, \zeta)$ are closely related to a set of interesting univalent analytic functions. In order to show this connection we complete the harmonic functions $G_{\varepsilon}(z, \zeta)$ to analytic functions in $z$. We will obtain, of course, two entirely different functions when $z$ lies in $D$ or $\tilde{D}$. Let us denote the analytic completion of $G_{\varepsilon}(z, \zeta)$ by $P_{\varepsilon}(z, \zeta)$ if $z \in D$ and by $\tilde{P}_{\varepsilon}(z, \zeta)$ if $z \in \tilde{D}$. We want to show that for fixed $\zeta \in D$

$$
f_{\varepsilon}(z)=e^{-1 / \varepsilon P_{\varepsilon}(z, \zeta)}, \tilde{f_{\varepsilon}^{*}}(z)=e^{-\widetilde{P}_{\varepsilon}(z, \zeta)}
$$

represent univalent analytic functions in $D$ and $\tilde{D}$, respectively.

For the sake of simplicity, we shall assume in the following consideration that $C$ is an analytic curve. There exists, therefore, an analytic function $z=f(t)$ which maps a neighborhood of a segment of the real axis in the $t$-plane onto a neighborhood of a given arc of $C$. The function $G_{\varepsilon}(z, \zeta)$ becomes a harmonic function $g(t)$ to both sides of the segment. It goes continuously through the segment, but its normal derivatives satisfy the discontinuity law

$$
\frac{\partial g}{\partial n}+\varepsilon \frac{\partial g}{\partial \tilde{n}}=0 \text { for real } t
$$

Let

$$
p(t)=P_{\varepsilon}(f(t), \zeta), \tilde{p}(t)=\tilde{P}_{\varepsilon}(f(t), \zeta) .
$$

We find easily for $t$ in the segment and in view of the described discontinuity behavior of $g(t)$ :

$$
\mathfrak{R}\left\{p^{\prime}(t)\right\}=\mathfrak{R}\left\{\tilde{p}^{\prime}(t)\right\}, \mathfrak{J}\left\{p^{\prime}(t)\right\}=\varepsilon \mathfrak{F}\left\{\tilde{p}^{\prime}(t)\right\} .
$$

We can combine the two relations (41) into the one equation:

$$
p^{\prime}(t)=\frac{1+\varepsilon}{2} \tilde{p}^{\prime}(t)+\frac{1-\varepsilon}{2} \tilde{p}^{\prime}(\bar{t}) .
$$

This formula allows an analytic continuation of $\tilde{p}^{\prime}(t)$ into the upper halfplane and of $p^{\prime}(t)$ into the lower. This proves that $p^{\prime}(t)$ and $\tilde{p}^{\prime}(t)$ are still analytic on the segment of the real axis in the $t$-plane. Re- 
turning to the $z$-plane we can infer that the functions

$$
P_{\varepsilon}^{\prime}(z, \zeta)=\frac{d}{d z} P_{\varepsilon}(z, \zeta), \quad \tilde{P}_{\varepsilon}^{\prime}(z, \zeta)=\frac{d}{d z} \tilde{P}_{\varepsilon}(z, \zeta)
$$

are analytic beyond the curve $C$. Thus we proved that the two determinations of the Green's functions $G_{\varepsilon}(z, \zeta)$ are still regular harmonic on $C$ if $C$ is an analytic curve.

We derive from (40) and (42) that

$$
\frac{P_{\varepsilon}^{\prime}(z, \zeta)}{\tilde{P}_{\varepsilon}^{\prime}(z, \zeta)}=\frac{1+\varepsilon}{2}+e^{-2 i \alpha} \frac{1-\varepsilon}{2}, \quad \alpha=\arg \tilde{p}^{\prime}(t) .
$$

Since we assume throughout $\varepsilon>0$, we see that the ratio (44) always lies in the right half of the complex plane. This implies

$$
\Delta \arg \tilde{P}_{\varepsilon}^{\prime}(z, \zeta)=\Delta \arg P_{\varepsilon}^{\prime}(z, \zeta)
$$

if $z$ runs through the curve $C$ in the positive sense with respect to $D$. But by the argument principle we have

$$
\Delta \arg P_{\varepsilon}^{\prime}(z, \zeta)=Z-P, \Delta \arg \tilde{P}_{\varepsilon}^{\prime}(z, \zeta)=\tilde{P}-\tilde{Z}
$$

where $P, Z$ are the numbers of zeros and poles of $P_{\varepsilon}^{\prime}$ in $D$ and $\tilde{P}, \tilde{Z}$ have the same meaning with respect to $\tilde{P}_{\varepsilon}^{\prime}$ and $\tilde{D}$. In case some zero of $P_{\varepsilon}^{\prime}$ should lie on $C$, we can deform the curve in such a way that it does not contain any zero and draw the same conclusion in view of the analyticity of $P_{\varepsilon}^{\prime}$ and $\tilde{P_{\varepsilon}^{\prime}}$ on $C$.

We know by definition that if $\zeta \in D$ we have

$$
P=1, Z \geq 0 ; \tilde{P}=0, \tilde{Z} \geq 1 \text {. }
$$

Hence, from (45), (46) and (47), we conclude

$$
Z-1 \leqq-1 \text {. }
$$

This is only possible if

$$
\tilde{Z}=1, Z=0 \text {. }
$$

Hence we can state that $P_{\varepsilon}^{\prime}(z, \zeta)$ and $\tilde{P}_{\varepsilon}^{\prime}(z, \zeta)$ do not vanish at any finite point of the $z$-plane.

Consider now the system of differential equations $(z=x+i y)$

$$
\frac{d x}{d t}=-\frac{\partial}{\partial x} G_{\varepsilon}(z, \zeta), \quad \frac{d y}{d t}=-\frac{\partial}{\partial y} G_{\varepsilon}(z, \zeta)
$$

Along each solution curve $x(t), y(t)$ of this system we have 


$$
\frac{d}{d t} G_{\varepsilon}(z(t), \zeta)=-\left[\left(\frac{d x}{d t}\right)^{2}+\left(\frac{d y}{d t}\right)^{2}\right]<0
$$

We have just shown that no critical point exists where $\nabla G_{\varepsilon}=0$. Hence the net of solution curves covers the entire $z$-plane in a regular manner. All curves start out from the point $z=\zeta$ and run towards infinity. Each curve possesses the integral

$$
\Im\{P(z, \zeta)\}=\text { const. or } \Im\left\{\tilde{P}_{\varepsilon}(z, \zeta)\right\}=\text { const. , }
$$

according as it is considered in $D$ or in $\tilde{D}$. From these facts it is evident that the functions (38) have the asserted univalency properties in $D$ and $\tilde{D}$, respectively.

The importance of our result lies in the fact that the numerous distortion theorems of univalent function theory are now at our disposal in order to derive estimates of the various potential theoretical quantities connected with $G_{\varepsilon}(z, \zeta)$ in terms of the geometry of the curve $C$.

Let us observe, further, that for $\varepsilon=1$ the function $\tilde{f}_{1}(z)$ represents the identity mapping while for $\varepsilon=0$ we conclude from (21) that

$$
\tilde{f_{0}}(z)=e^{-\tilde{\gamma}} \cdot e^{\tilde{P}(z, \infty)}=z+c_{1}+\frac{c_{1}}{z}+\cdots
$$

is the univalent function which maps $\check{D}$ onto the exterior of a circle of radius $e^{-\bar{\gamma}}$ and which has at infinity the derivative one. Thus we can interpolate a continuous sequence of univalent mappings between the identity map of $\tilde{D}$ and its normalized mapping onto the exterior of a circle.

The preceding considerations show clearly the significance of the Fredholm eigen values and eigen functions for the dielectric problem and the general potential theory of the curve $C$. A generalization of most concepts to the physically more interesting case of three dimensions is easily done.

3. The variation of the eigen values. The variation of the eigen values $\lambda_{\nu}$ under a variation of the curve $C$ can be determined by using the variational theory of the Green's function and of the various kernel functions connected with it [3]. In this paper we wish to give a straightforward and elementary derivation of the variational formulas.

Let $z_{0}$ be an arbitrary fixed point in $\tilde{D}$ and consider the mapping

$$
z^{*}=z+\frac{\alpha}{z-z_{0}} .
$$


For small enough $\alpha$ this will be a univalent mapping of $C$ into a new smooth curve $C^{*}$. Let us denote its eigen values by $\lambda_{\nu}^{*}$ and its eigen functions by $w_{\nu}^{*}(z)$. We have used various eigen function definitions in the domain $D$; the $w_{\nu}^{*}(z)$ shall play the same role with respect to $D^{*}$ (the domain bounded by $C^{*}$ ) as the $w_{\nu}(z)$ defined in Section 1 played with respect to $D$.

We have the integral equation

$$
w_{\nu}^{*}\left(z^{*}\right)=\frac{\lambda_{\nu}^{*}}{2 \pi i} \int_{\sigma^{*}} \frac{\left(\overline{\left.w_{\nu}^{*}\left(\zeta^{*}\right) d \zeta^{*}\right)}\right.}{\zeta^{*}-z^{*}}, \quad z^{*} \in D^{*}
$$

Let us define

$$
m_{\nu}(z)=w_{\nu}^{*}\left(z+\frac{\alpha}{z-z_{3}}\right)\left(1-\frac{\alpha}{\left(z-z_{3}\right)^{2}}\right) .
$$

This is a regular analytic function in $D$ since (1) maps $D$ univalently onto $D^{*}$ where $w_{\nu}^{*}\left(z^{*}\right)$ is analytic. Using (3), we can rewrite (2) into the simpler form

$$
m_{\nu}(z)=\left(1-\frac{\alpha}{\left(z-z_{0}\right)^{2}}\right) \cdot \frac{\lambda_{\nu}^{*}}{2 \pi i} \int_{c}\left[1-\frac{\alpha}{\left(z-z_{0}\right)\left(\zeta-z_{0}\right)}\right]^{-1} \frac{\left(m_{\nu}(\zeta) d \zeta\right)}{\zeta-z} .
$$

We have thus referred all variables back to our original domain $D$, but $\lambda_{\nu}^{*}$ and $m_{\nu}(z)$ appear now as the eigen values and eigen functions of an integral equation with slightly changed kernel.

We may transform the new integral equation (4) by easy calculations into

$$
m_{\nu}(z)=\frac{\lambda_{\nu}^{*}}{\pi} \iint_{D} \frac{m_{v}(\zeta)}{(\zeta-z)^{2}} d \tau-\alpha \frac{\lambda_{\nu}^{*}}{\pi} \iint_{D\left[\left(z-z_{0}\right)\left(\zeta-z_{0}\right)-\alpha\right]^{2}} \cdot \frac{m_{\nu}(\zeta) d \tau}{}
$$

Observe that by the definition (3) we have

$$
\iint_{D}\left|m_{\nu}(z)\right|^{2} d \tau=\iint_{D *}\left|w_{\nu}^{*}\left(z^{*}\right)\right|^{2} d \tau^{*}=1
$$

We have thus to determine the normalized eigen functions $m_{\nu}(z)$ to the integral equation (5) which differs from our original equation (1.13) by an $\alpha$-term which can be estimated uniformly in $z$ for $z_{0} \in \tilde{D}$ fixed.

Let us define the analytic function [3]

$$
L(z, \zeta)=-\frac{2}{\pi} \frac{\partial^{2}}{\partial z \partial \zeta} G(z, \zeta)=\frac{1}{\pi(z-\zeta)^{2}}-l(z, \zeta) .
$$

It is well-known that for every function $f(z)$ which is analytic in $D$ and for which $\iint_{D}|f|^{2} d \tau<\infty$ holds 


$$
\iint_{D} L(z, \zeta) \overline{f(\zeta)} d \tau=0
$$

Hence we have the identity, valid for each such $f(z)$,

$$
\frac{1}{\pi} \iint_{D} \frac{\overline{f(\zeta)}}{(\zeta-z)^{2}} d \tau=\iint_{D} l(z, \zeta) \overline{f(\zeta)} d \tau
$$

Under our assumptions about the boundary curve $C$ of $D$, it can be shown that $l(z, \zeta)$ is continuous in both variables in the closed region $D+C$. Thus (5) can be put into the form:

$$
m_{\nu}(z)=\lambda_{\nu}^{*} \iint_{D} l\left(z, \zeta \overline{m_{\nu}(\zeta)} d \tau-\alpha \frac{\lambda_{\nu}^{*}}{\pi} \iint_{D} \frac{\overline{m_{\nu}(\zeta)}}{\left[\left(z-z_{0}\right)\left(\zeta-z_{0}\right)-\alpha\right]^{2}} d \tau,\right.
$$

while $w_{\nu}(z)$ satisfies the unperturbed integral equation

$$
w_{\nu}(z)=\lambda_{\nu} \iint_{D} l(z, \zeta) \overline{w_{\nu}(\zeta) d \tau}
$$

Now we can apply the general perturbation theory for regular kernels [9] and state that the eigen functions $m_{\nu}(z)$ and the eigen values $\lambda_{\nu}^{*}$ are analytic functions of the perturbation parameters $\alpha$ and $\bar{\alpha}$ and can be developed in power series in them. For $\alpha=0, \lambda_{\nu}^{*}$ will coincide with $\lambda_{\nu}$ while $m_{\nu}(z)$ will then lie in the linear space spanned by the eigen functions of (11) which belong to the unperturbed eigen value $\lambda_{2}$.

Let $w_{\nu}^{(j)}(z)(j=1, \cdots, n)$ denote the eigen functions belonging to $\lambda_{\nu}$. We have the developments

$$
\lambda_{\nu}^{*}=\lambda_{\nu}+|\alpha| \kappa_{\nu}+O\left(|\alpha|^{2}\right)
$$

and

$$
m_{\nu}(z)=\sum_{j=1}^{n} A_{j} w_{\nu}^{(j)}(z)+|\alpha| \omega_{\nu}(z)+O\left(|\alpha|^{2}\right) .
$$

Inserting (12) and (13) into (10) and making use of (11), we find

$$
\begin{aligned}
\sum_{j=1}^{n} A_{j} w_{\nu}^{(j)}(z)= & \frac{\lambda_{\nu}^{*}}{\lambda_{\nu}} \sum_{j=1}^{n} \bar{A}_{j} w_{\nu}^{(j)}(z)+|\alpha| \lambda_{\nu} \iint_{D} l(z, \zeta) \overline{\omega_{\nu}(\zeta)} d \tau \\
& -|\alpha| \omega_{\nu}(z)-\frac{\alpha \lambda_{\nu}}{\left(z-z_{0}\right)^{2} \pi} \sum_{j=1}^{n} \bar{A}_{j} \iint_{D} \frac{\overline{w_{\nu}^{(j)}(\zeta)}}{\left(\zeta-z_{0}\right)^{2}} d \tau+O\left(|\alpha|^{2}\right) .
\end{aligned}
$$

We multiply this identity with $w_{\nu}^{(k)}(z)$ and integrate over $D$. We use the orthonormality of the $w_{\nu}^{(k)}(z)$, the symmetry of $l(z, \zeta)$ and the integral equation (11). We also make use of the fact that by (1.36) 


$$
\frac{1}{\pi} \iint_{D} \frac{\overline{w_{\nu}(\zeta)}}{\left(\zeta-z_{0}\right)^{2}} d \tau=\frac{\sqrt{\lambda_{\nu}^{2}-1}}{i \lambda_{\nu}} \tilde{w}_{\nu}\left(z_{0}\right)
$$

Hence we arrive at

$$
\begin{aligned}
A_{k}= & \frac{\lambda_{\nu}^{*}}{\lambda_{\nu}} \bar{A}_{k}+2 i|\alpha| \Im\left\{\iint_{D} w_{\nu}^{(k)}(z) \overline{\omega_{\nu}(z)} d \tau\right\} \\
& +\alpha \frac{\pi\left(\lambda_{\nu}^{2}-1\right)}{\lambda_{\nu}} \sum_{j=1}^{n} \bar{A}_{k} \tilde{w}_{\nu}^{(j)}\left(z_{0}\right) \tilde{w}_{\nu}^{(k)}\left(z_{0}\right)+O\left(|\alpha|^{2}\right), \quad k=1,2, \cdots, n .
\end{aligned}
$$

Using the development (12) and comparing equal powers of $|\alpha|$ on both sides, we obtain

$$
\Im\left\{A_{k}\right\}=0, \quad A_{k}=\text { real, } k=1,2, \cdots, n .
$$

Taking real parts in (16) and putting

$$
\operatorname{sgn} \alpha=\frac{\alpha}{|\alpha|}=e^{i \beta},
$$

we find

$$
\kappa_{\nu} A_{k}+\pi\left(\lambda_{\nu}^{2}-1\right) \sum_{j=1}^{n} A_{j} \Re\left\{e^{i \beta} \tilde{w}_{\nu}^{(j)}\left(z_{0}\right) \tilde{w}_{\nu}^{(k)}\left(z_{0}\right)\right\}=0, \quad k=1,2, \cdots, n .
$$

Thus the possible values of $\kappa_{\nu}$ in the development (12) of the perturbed eigen value $\lambda_{\nu}^{*}$ are the eigen values of the secular equation

$$
\operatorname{det}\left\|\kappa_{\nu} \delta_{j k}+\pi\left(\lambda_{\nu}^{2}-1\right) \Re\left\{e^{i \beta} \tilde{w}_{\nu}^{(j)}\left(z_{0}\right) \tilde{w}_{\nu}^{(k)}\left(z_{0}\right)\right\}\right\|=0 .
$$

In particular, if $\lambda_{\nu}$ is a simple (nondegenerate) eigen value, we have the simple variational formula

$$
\delta \lambda_{\nu}=|\alpha| \cdot \kappa_{\nu}=-\pi\left(\lambda_{\nu}^{2}-1\right) \Re\left\{\alpha \tilde{w}_{\nu}\left(z_{0}\right)^{2}\right\} .
$$

Let us suppose next that we perform a variation (1) of the curve $C$ but now with $z_{0} \in D$. Since the mapping (1) is regular and univalent in $\tilde{D}$, we can repeat the entire argument by interchanging the roles of $D$ and $\tilde{D}$. We thus find

$$
\operatorname{det}\left\|\kappa_{\nu} \delta_{j k}+\pi\left(\lambda_{\nu}^{2}-1\right) \Re\left\{e^{i \beta} w_{\nu}^{(j)}\left(z_{0}\right) w_{\nu}^{(k)}\left(z_{0}\right)\right\}\right\|=0
$$

as the secular equation for the $\kappa_{2}$-terms and

$$
\delta \lambda_{\nu}=|\alpha| \kappa_{\nu}=-\pi\left(\lambda_{\nu}^{2}-1\right) \Re\left\{\alpha w_{\nu}\left(z_{0}\right)^{2}\right\}
$$

in the nondegenerate case. Formulas (21) and (23) exhibit the complete symmetry of our theory with respect to $D$ and $\tilde{D}$. 
We used the method of interior variations (1) in order to reduce the variational problem for the $\lambda_{\nu}$ explicitly to the theory of perturbation in classical integral equation theory. The formulas obtained are also very convenient in various extremum problems regarding the $\lambda_{2}$ as we shall show later. It seems, however, desirable to give also a variational formula for deformations of $C$ which are described by the normal shift $\delta n$ of each point on $C$. For this purpose we put

$$
\begin{aligned}
& \Re\left\{\pi\left(\lambda_{\nu}^{2}-1\right) \alpha w_{\nu}^{(j)}\left(z_{\jmath}\right) w_{\nu}^{(k)}\left(z_{0}\right)\right\} \\
& \quad=\Re\left\{\frac{\alpha}{2 i}\left(\lambda_{\nu}^{2}-1\right) \oint_{C} \frac{w_{\nu}^{(j)}(\zeta) w_{\nu}^{(k)}(\zeta)}{\zeta-z_{0}} d \zeta\right\}, \quad z_{\jmath} \in D .
\end{aligned}
$$

Applying Cauchy's integral theorem with respect to $\tilde{D}$, we also find

$$
0=\Re\left\{\frac{\alpha}{2 i}\left(\lambda_{\nu}^{2}-1\right) \oint_{C} \frac{\tilde{w}_{\nu}^{(j)}(\zeta) \tilde{w}_{\nu}^{(k)}(\zeta)}{\zeta-z_{J}} d \zeta\right\} .
$$

Finally, we derive from (1.34) that

$$
\begin{aligned}
\left(\lambda_{\nu}^{2}-1\right) & \left\{w_{\nu}^{(j)}(\zeta) w_{\nu}^{(k)}(\zeta)-\tilde{w}_{\nu}^{(j)}(\zeta) \tilde{w}_{\nu}^{(k)}(\zeta)\right\} \zeta^{\prime 2} \\
& =2 \Re\left\{\lambda_{\nu} \tilde{w}_{\nu}^{(j)}(\zeta) \overline{\tilde{w}_{\nu}^{(k)}(\zeta)}-\lambda_{\nu}^{2} \tilde{w}_{\nu}^{(j)}(\zeta) \tilde{w}_{\nu}^{(k)}(\zeta) \zeta^{\prime 2}\right\} .
\end{aligned}
$$

Hence, if we subtract (25) from (24), we obtain

$$
\begin{aligned}
\Re & \left.\pi\left(\lambda_{\nu}^{2}-1\right) \alpha w_{\nu}^{(j)}\left(z_{0}\right) w_{\nu}^{(k)}\left(z_{0}\right)\right\} \\
\quad & \oint_{C} \Re\left\{\lambda_{\nu} \tilde{w}_{\nu}^{(j)}(\zeta) \overline{\tilde{w}_{\nu}^{(k)}(\zeta)}-\lambda_{\nu}^{2} \tilde{w}^{(j)}(\zeta) \tilde{w}_{\nu}^{(k)}(\zeta) \zeta^{\prime 2}\right\} \delta n d s
\end{aligned}
$$

where

$$
\delta n=\Re\left\{\frac{1}{i \zeta^{\prime}} \frac{\alpha}{\zeta-z_{0}}\right\}
$$

represents the normal shift of $C$ under the deformation (1). Thus the coefficients of the secular equation for $\delta \lambda$ have been expressed in terms of $\delta n$.

In particular, we have in the nondegenerate case in view of (23)

$$
\delta \lambda_{\nu}=\int_{C}\left[\lambda_{\nu}^{2} \Re\left\{\tilde{w}_{\nu}(\zeta)^{2} \zeta^{\prime 2}\right\}-\lambda_{\nu}\left|\tilde{w}_{\nu}(\zeta)\right|^{2}\right] \delta n d s .
$$

It can easily be verified from (1.34) that on $C$

$$
\begin{aligned}
\lambda_{\nu} \Re\left\{\tilde{w}_{\nu}^{(j)} \tilde{w}_{\nu}^{(k)}\right\}-\lambda_{\nu}^{2} \Re\left\{\tilde{w}_{\nu}^{(j)} \tilde{w}_{\nu}^{(k)} \zeta^{\prime 2}\right\} \\
\quad=-\lambda_{\nu} \Re\left\{w_{\nu}^{(j)} \overline{w_{\nu}^{(k)}}\right\}+\lambda_{\nu}^{2} \Re\left\{w_{\nu}^{(j)} w_{\nu}^{(k)} \zeta^{\prime 2}\right\} .
\end{aligned}
$$

Thus we may replace $\tilde{w}$ by $w$ in formulas (27) and (29); since transition 
from $D$ to $\tilde{D}$ implies also a change of sign of the interior normal, the end result is unchanged. Thus the variational formula of the Hadamard type (29) is entirely symmetric with respect to the two complementary domains considered. If we had chosen $z_{0} \in \tilde{D}$, we would have obtained the same end result (29).

We derived (29) in the case of a particular variation of the type (1). But since a variational formula depends linearly and additively on the variation, and since we can approximate general $\delta n$-variations by superposition of special variations of the type (1), we can extend (29) to the most general case of a $\delta n$-variation.

The value of the variational formula (29) is of heuristic nature; it shows the dependence of $\lambda_{2}$ on the geometry of $C$. For a precise study of extremum problems it is preferable to apply the variational formulas based on interior variations of the type (1).

We can derive, however, interesting monotonicity results by means of (29). Let, for example, $z=f(u)$ give the conformal mapping of the unit circle $|u|<1$ onto the domain $D$. Let $C_{r}$ be the image under this map of the circumference $|u| \approx r<1$; and let $\lambda(r), w(z, r)$ denote, say, the 2 th eigen value and eigen function of $C_{r}$. We assume, for the sake of simplicity, that $\lambda(r)$ is nondegenerate and then easily derive from (29):

$$
\begin{aligned}
\frac{d}{d r} \lambda(r)= & -\lambda(r) \oint_{|u|=r}|w(z, r)|^{2}\left|f^{\prime}(u)\right|^{2} d s_{u} \\
& +\lambda(r)^{2} \Re\left\{\frac{i}{r} \oint_{|u|=r} w(z, r)^{2} f^{\prime}(u)^{2} u d u\right\} .
\end{aligned}
$$

The function

$$
\left.F_{r}(u)=w[f(u), r)\right] f^{\prime}(u)
$$

is regular analytic for $|u| \leqq r$; hence the second integral in $\left(29^{\prime}\right)$ vanishes by Cauchy's integral theorem and we obtain:

$$
\frac{d}{d r} \log \lambda(r)=-\int_{|u|=r}\left|F_{r}(u)\right|^{2} d s_{u b}<0 .
$$

The eigen values $\lambda(r)$ of the level curves $C_{r}$ are monotonically decreasing if $r$ increases.

For every function $F(u)$ which is regular analytic for $|u| \leqq r$ holds the obvious inequality

$$
\int_{|u|=r}|F(u)|^{2} d s_{u} \geqq \frac{2}{r} \iint_{|u|<r}|F(u)|^{2} d \tau .
$$


Observe now that because of the normalization of $w(z, r)$ inside of $C_{r}$ the function $F_{r}(u)$ is normalized in the circle $|u|<r$. Hence, combining $\left(29^{\prime \prime}\right)$ with $\left(29^{\prime \prime \prime}\right)$, we finally obtain

$$
\frac{d}{d r}\left(\lambda r^{2}\right) \leqq 0
$$

Since we have the trivial estimate $\lambda(1) \geqq 1$ for every curve $C$, we then derive from $\left(29^{\text {iv }}\right)$ the useful estimate

$$
\lambda(r) \geqq \frac{1}{r^{2}} \quad \text { for } r \leqq 1
$$

In order to apply the usual perturbation method of integral equation theory we had to replace the integral equation (1.13) with singular kernel by the integral equation (11) which has the regular symmetric kernel $l(z, \zeta)$. The necessity for this transition becomes clear when we consider the exceptional case that $C$ is a circumference. In this case (and only then), we have $l(z, \zeta)=0$. The original integral equation (1.13) has only the eigen value $\lambda=\infty$ and each function $f(z)$ which is analytic in $D$ is an eigen function.

In fact, suppose for the sake of simplicity that $C$ is the unit circumference $z \cdot \bar{z}=1$. We have

$$
\frac{1}{\pi} \iint_{|\zeta|<1} \frac{\overline{f(\zeta)}}{(\zeta-z)^{2}} d \tau=\frac{1}{2 \pi i} \oint_{|\zeta|=1} \frac{\overline{f(\zeta) d \zeta}}{\zeta-z}=\frac{1}{2 \pi i} \oint_{|\zeta|=1} \frac{\zeta f(\zeta) d \zeta}{\zeta \bar{z}-1}
$$

By means of the residue theorem we conclude therefore

$$
\frac{1}{\pi} \iint_{|\zeta|<1} \frac{\overline{f(\zeta)}}{(\zeta-z)^{2}} d \tau= \begin{cases}0 & \text { if }|z|<1 \\ \frac{1}{z^{2}} \bar{f}\left(\frac{1}{z}\right) & \text { if }|z|>1 .\end{cases}
$$

This equation proves our statement that $\lambda=\infty$ is the only eigen value of (1.13) in this case and that it is of infinite degeneracy.

Our variational theory does not work in this exceptional case. However, let $\left|z_{0}\right|>1$ and $C^{*}$ be the image of $|z|=1$ under the variation (1). We define its eigen function $w_{\nu}^{*}(z)$ and by (3) a function $m_{\nu}(z)$ which is regular analytic in $D$. It satisfies the integral equation (5) which, in view of (32), can be brought into the simple form

$$
m_{\nu}(z)=-\frac{\alpha \lambda_{\nu}^{*}}{\left(z-z_{0}\right)^{2}} \frac{1}{\eta^{2}} \bar{m}_{\nu}\left(\frac{1}{\eta}\right), \quad \eta=z_{0}+\frac{\alpha}{z-z_{0}} .
$$




$$
m_{\nu}(z)=\frac{d}{d z} M_{\nu}(z)
$$

if we choose the right constant of integration in the definition of $M_{\nu}(z)$, we can integrate (33) to the identity

$$
M_{\nu}(z)=-\lambda_{\nu}^{*} \bar{M}_{\nu}(L(z)),
$$

where

$$
L(z)=\eta^{-1}=\frac{z-z_{0}}{z_{0}\left(z-z_{0}\right)+\alpha}
$$

is a linear function of $z$. Thus we obtain a simple functional equation for the eigen functions $w_{\nu}^{*}(z)$ and the eigen values $\lambda_{\nu}^{*}$ of the varied curve $C^{*} . \quad \alpha$ must be sufficiently small in order that the mapping (1) be univalent in $D$; but we have not made any neglection of higher powers of $\alpha$ and (35) will give the precise value of $\lambda_{2}^{*}$.

If we iterate (35), we obtain

$$
M_{\nu}(z)=\lambda_{\nu}^{* 2} M_{\nu}(\Lambda(z)), \quad \Lambda=\bar{L}(L(z)) .
$$

If $z_{1}, z_{2}$ are the fixed points of the linear transformation $Z=\Lambda(z)$, we can write

$$
\frac{Z-z_{1}}{Z-z_{2}}=\tau^{2} \frac{z-z_{1}}{z-z_{2}}
$$

where $\left|z_{1}\right|<1,\left|z_{2}\right|>1$. The eigen functions $M_{\nu}(z)$ are of the form

$$
M_{\nu}(z)=A_{\nu}\left(\frac{z-z_{1}}{z-z_{2}}\right)^{\nu}, \quad \quad \nu=1,2 \cdots
$$

and belong to the eigen values

$$
\lambda_{\nu}^{*}= \pm \tau^{-\nu}
$$

Thus all eigen functions and eigen values of the curve $C^{*}$ can be calculated explicitly. An easy computation shows that for small values of $\varepsilon$

$$
\tau^{-1}=\frac{\left(\left|z_{0}\right|^{2}-1\right)^{2}}{|\alpha|}+O(1)
$$

An analogous calculation can be performed if the unit circle is transformed by a variation (1) with $\left|z_{0}\right|<1$. If we consider a superposition of variations (1), we can still derive an asymptotic formula for the eigen values $\lambda_{\nu}^{*}$ obtained. Thus we have shown that the eigen values for nearly circular domains can be obtained asymptotically in 
spite of the fact that the circle has an infinitely degenerate eigen value.

We showed at the end of $\S 1$ that the eigen values of an ellipse can be calculated explicitly. This result is a particular case of our preceding investigation since the exterior of the ellipse is obtained from the exterior of the unit circle by a transformation (1) with $z_{0}=0$ and $|\alpha|<1$.

There are relatively few domains for which the eigen values and eigen functions of the Fredholm integral equation are known. It is, therefore, important to possess at least an asymptotic formula for the eigen values of nearly circular domains which admits many arbitrary parameters. Such formulas are particularly useful when one wishes to test hypotheses with respect to the eigen values of general domains.

4. The variation of the dielectric Green's function. In this section we want to derive the formula for the variation of the dielectric Green's function $G_{\varepsilon}(z, \zeta)$ defined in $\S 2$. It will appear that it possesses a very simple variational formula which is quite similar to that for the ordinary Green's function of a plane domain. We shall again consider the interior variation

$$
z^{*}=z+\frac{\alpha}{z-z_{0}}
$$

which transforms the curve $C$ into a curve $C^{*}$ defining the two complementary domains $D^{*}$ and $\tilde{D}^{*}$. Let $G_{\varepsilon}^{*}(z, \zeta)$ be the corresponding dielectric Green's function to the parameter $\varepsilon$.

If $z_{0} \in \tilde{D}$, the mapping (1) will be univalent and regular in $D$ for small enough $\alpha$; hence the function

$$
\Gamma_{\varepsilon}(z, \zeta)=G_{\varepsilon}^{*}\left(z+\frac{\alpha}{z-z_{0}}, \zeta+\frac{\alpha}{\zeta-z_{0}}\right)
$$

will be harmonic in $D$. It will also be harmonic in $\tilde{D}$, except for the interior of a circle of radius $|\alpha|^{1 / 2}$ around the point $z_{0}$. The function $\Gamma_{\varepsilon}(z, \zeta)$ will have logarithmic poles at infinity and for $z=\zeta$ as follows from the definition of $G_{\varepsilon}(z, \zeta)$.

We consider now Green's identity :

$$
\begin{array}{r}
\frac{1}{2 \pi} \int_{C}\left[\Gamma_{\varepsilon}(t, z) \frac{\partial G_{\varepsilon}(t, \zeta)}{\partial n}-G_{\varepsilon}(t, \zeta)^{\partial \Gamma_{\varepsilon}(t, z)} \frac{\partial n}{\partial n}\right] d s \\
=\varepsilon \Gamma_{\varepsilon}(\zeta, z) \delta(\zeta)-\varepsilon G_{\varepsilon}(z, \zeta) \delta(z) .
\end{array}
$$

Observe that in view of the conformality of (1) on $C$ the function $\Gamma_{\varepsilon}(z, \zeta)$ has the same continuity (and discontinuity) property on $C$ as 
the original function $G_{\varepsilon}(z, \zeta)$. Hence we may transform (3) into

$$
\begin{gathered}
\varepsilon\left[\Gamma_{\varepsilon}(\zeta, z) \delta(\zeta)-G_{\varepsilon}(z, \zeta) \delta(z)\right]=-\frac{\varepsilon}{2 \pi} \int_{C}\left[\Gamma_{\varepsilon}(t, z) \frac{\partial G_{\varepsilon}(t, \zeta)}{\partial \tilde{n}}\right. \\
\left.-G_{\varepsilon}(t, \zeta) \frac{\partial \Gamma_{\varepsilon}(t, z)}{\partial \tilde{n}}\right] d s .
\end{gathered}
$$

Now we can apply Green's identity with respect to the domain $\tilde{D}$ after removing from it the interior of the circle $\left|z-z_{0}\right|=|\alpha|^{1 / 2}$ which we denote by $c$. Let us assume that neither $z$ nor $\zeta$ lie inside $c$; then (4) yields

( 5$) \quad \Gamma_{\varepsilon}(z, \zeta)-G_{\varepsilon}(z, \zeta)=-\frac{1}{2 \pi} \int_{c}\left[\Gamma_{\varepsilon}(t, z) \frac{\partial G_{\varepsilon}(t, \zeta)}{\partial n}-G_{\varepsilon}(t, \zeta) \frac{\partial \Gamma_{\varepsilon}(t, z)}{\partial n}\right] d s$.

We have now fully utilized the boundary behavior of $G_{\varepsilon}(z, \zeta)$. The evaluation of the $c$-integral follows exactly the lines of the calculation for the ordinary Green's function. We put for $t \in c$

$$
t=z_{0}+|\alpha|^{1 / 2} e^{i \phi}
$$

and evaluate the right-hand integral in (5) by power series development. We define again two analytic functions of $z$, namely $p_{\varepsilon}(z, \zeta)$ and $p_{\varepsilon}^{*}(z, \zeta)$, by

$$
\Re\left\{P_{\varepsilon}(z, \zeta)\right\}=G_{\varepsilon}(z, \zeta), \quad \Re\left\{P_{\varepsilon}^{*}(z, \zeta)\right\}=G_{\varepsilon}^{*}(z, \zeta) .
$$

Further, let

$$
P_{\varepsilon}^{\prime}(z, \zeta)=\frac{d}{d z} P_{\varepsilon}(z, \zeta), P_{\varepsilon}^{* \prime}(z, \zeta)=\frac{d}{d z} P_{\varepsilon}^{*}(z, \zeta)
$$

Then the usual calculations yield

$$
G_{\varepsilon}^{*}\left(z^{*}, \zeta^{*}\right)-G_{\varepsilon}(z, \zeta)=\Re\left\{\alpha P_{\varepsilon}^{*^{\prime}}\left(z_{0}, z^{*}\right) P_{\varepsilon}^{\prime}\left(z_{0}, \zeta\right)\right\}+O\left(|\alpha|^{2}\right) .
$$

Further series development leads to the simple result

$$
\begin{aligned}
G_{\varepsilon}^{*}(z, \zeta)=G_{\varepsilon}(z, \zeta) & +\Re\left\{\alpha \left[P_{\varepsilon}^{\prime}\left(z_{0}, z\right) P_{\varepsilon}^{\prime}\left(z_{0}, \zeta\right)\right.\right. \\
& \left.\left.-\frac{P_{\varepsilon}^{\prime}(z, \zeta)}{z-z_{0}}-\frac{P_{\varepsilon}^{\prime}(\zeta, z)}{\zeta-z_{0}}\right]\right\}+O\left(|\alpha|^{2}\right) .
\end{aligned}
$$

This is exactly the same variational formula as for the ordinary Green's function $[12,13]$. It has been derived for $z_{0} \in \tilde{D}$.

If we had chosen $z_{0} \in D$ instead of $\tilde{D}$ analogous calculations would have been applicable. We could start with 


$$
\begin{aligned}
\frac{1}{2 \pi} \int_{C}\left[\Gamma_{\varepsilon}(t, z) \frac{\partial G_{\varepsilon}(t, \zeta)}{\partial \tilde{n}}\right. & \left.-G_{\varepsilon}(t, \zeta) \frac{\partial \Gamma_{\varepsilon}(t, z)}{\partial \tilde{n}}\right] d s \\
= & \Gamma_{\varepsilon}(\zeta, z) \tilde{\delta}(\zeta)-G_{\varepsilon}(z, \zeta) \tilde{\delta}(z) .
\end{aligned}
$$

Using the discontinuity of $\frac{\partial G_{\varepsilon}}{\partial n}$ on $C$, we find

$$
\begin{aligned}
\varepsilon\left[\Gamma_{\varepsilon}(\zeta, z) \tilde{\delta}(\zeta)-G_{\varepsilon}(z, \zeta) \tilde{\delta}(z)\right]=-\frac{1}{2 \pi} \int_{C}\left[\Gamma_{\varepsilon}(t, z) \frac{\partial G_{\varepsilon}(t, \zeta)}{\partial n}\right. & \\
& \left.-G_{\varepsilon}(t, \zeta) \frac{\partial \Gamma_{\varepsilon}(t, z)}{\partial n}\right] d s
\end{aligned}
$$

and by means of Green's identity

$$
\varepsilon\left[\Gamma_{\varepsilon}(z, \zeta)-G_{\varepsilon}(z, \zeta)\right]=-\frac{1}{2 \pi} \int_{c}\left(\Gamma_{\varepsilon} \frac{\partial G_{\varepsilon}}{\partial n}-G_{\varepsilon} \frac{\partial \Gamma_{\varepsilon}}{\partial n}\right) d s
$$

where $c$ denotes again the circle $\left|z-z_{0}\right|=|\alpha|^{1 / 2}$. In this case the same procedure as before yields the result for $z_{0} \in D$ :

$$
\begin{aligned}
G_{\varepsilon}^{*}(z, \zeta)-G_{\varepsilon}(z, \zeta)=\Re\left\{\alpha \left[\frac{1}{\varepsilon}\right.\right. & P_{\varepsilon}^{\prime}\left(z_{0}, z\right) P_{\varepsilon}^{\prime}\left(z_{0}, \zeta\right) \\
& \left.\left.-\frac{P_{\varepsilon}^{\prime}(z, \zeta)}{z-z_{0}}-\frac{P_{\varepsilon}^{\prime}(\zeta, z)}{\zeta-z_{0}}\right]\right\}+O\left(|\alpha|^{2}\right) .
\end{aligned}
$$

Observe the factor $\frac{1}{\varepsilon}$ which is now introduced into (13) and causes a slight change in the variational formula.

We have thus derived a very elegant variational formula for the dielectric Green's function; its significance is seen from the numerous applications of its analogue in the case of the ordinary Green's function $[12,13,14]$.

As mentioned in $\S 2$, the function $P_{\varepsilon}(z, \zeta)$ consists in reality of two analytic functions, say, $P_{\varepsilon}(z, \zeta)$ if $z \in D$ and $\tilde{P}_{\varepsilon}(z, \zeta)$ if $z \in \tilde{D}$. The boundary behavior of $G_{\varepsilon}(z, \zeta)$ as described in $\S 2$ implies for $z \in C$

$$
\Re\left\{P_{\varepsilon}^{\prime}(z, \zeta) z^{\prime}\right\}=\Re\left\{\tilde{P}_{\varepsilon}^{\prime}(z, \zeta) z^{\prime}\right\}, \quad \Im\left\{P_{\varepsilon}^{\prime}(z, \zeta) z^{\prime}\right\}=\varepsilon \mathfrak{F}\left\{\tilde{P}_{\varepsilon}^{\prime}(z, \zeta) z^{\prime}\right\} .
$$

We can combine the variational formulas (9) and (13) into the integral form :

$$
\begin{aligned}
& G_{\varepsilon}^{*}(z, \zeta)-G_{\varepsilon}(z, \zeta) \\
& \quad=\Re\left\{\frac{\alpha}{2 \pi i} \oint_{\sigma} \frac{\frac{1}{\varepsilon} P_{\varepsilon}^{\prime}(t, z) P_{\varepsilon}^{\prime}(t, \zeta)-\tilde{P}_{\varepsilon}(t, z) \tilde{P}_{\varepsilon}^{\prime}(t, \zeta)}{t-z_{0}} d t\right\}+O\left(|\alpha|^{2}\right) .
\end{aligned}
$$


By use of (14) this can be simplified to

$$
\begin{aligned}
& \delta G_{\varepsilon}(z, \zeta) \\
= & \frac{1}{2 \pi}\left(\frac{1}{\varepsilon}-1\right) \int_{\sigma}\left[\frac{\partial G_{\varepsilon}(t, z)}{\partial s} \frac{\partial G_{\varepsilon}(t, \zeta)}{\partial s}-\frac{\partial G_{\varepsilon}(t, z)}{\partial n} \frac{\partial G_{\varepsilon}(t, \zeta)}{\partial \tilde{n}}\right] \delta n d s
\end{aligned}
$$

with

$$
\delta n=\Re\left\{\frac{1}{i t^{\prime}} \frac{\alpha}{\left(t-z_{0}\right)}\right\}
$$

This is the Hadamard type variational formula for the dielectric Green's function which has been proved in a precise manner through use of our interior variational method.

Since we can also write (16) in the form

$$
\begin{aligned}
& \delta G_{\varepsilon}(z, \zeta) \\
= & \frac{1}{2 \pi}\left(\frac{1}{\varepsilon}-1\right) \int_{C}\left[\frac{\partial G_{\varepsilon}(t, z)}{\partial s} \frac{\partial G_{\varepsilon}(t, \zeta)}{\partial s}+\frac{1}{\varepsilon} \frac{\partial G_{\varepsilon}(t, z)}{\partial n}-\frac{\partial G_{\varepsilon}(t, \zeta)}{\partial n}\right] \text { in } d s
\end{aligned}
$$

it is evident that if $\zeta \in D$ the expression $\left(G_{\varepsilon}(z, \zeta)+\varepsilon \log |z-\zeta|\right)_{z=\zeta}$ depends monotonically upon the domain $D$ while for $\zeta \in \tilde{D}$ the same is true for $\left(G_{\varepsilon}(z, \zeta)+\log |z-\zeta|\right)_{z=\zeta}$. In a similar way many other expressions can be constructed which have a definite factor of $\delta n d s$ under the integral sign and which depend, therefore, monotonically upon $D$. The application of Hadamard's formula in order to obtain inequalities and comparison theorems for functionals connected with $G_{\varepsilon}(z, \zeta)$ is obvious.

For $\varepsilon=1$, we have $G_{\varepsilon}(z, \zeta)=-\log |z-\zeta|$ independently of the domain. For this reason the factor $\left(\frac{1}{\varepsilon}-1\right)$ must occur in the variational formulas (16) and (18).

We showed at the end of $\S 2$ that the mapping of a domain onto a circle can be connected with the identical mapping by a one-parameter family of univalent functions which are closely related to the dielectric Green's functions. For this reason it is of interest to compute the derivative of $G_{\varepsilon}(z, \zeta)$ with respect to $\varepsilon$.

We start with Green's identity and with $\varepsilon>0, e>0$ :

$$
\begin{aligned}
e \delta(\zeta) G_{\varepsilon}(\zeta, & \eta)-\varepsilon \delta(\eta) G_{e}(\zeta, \eta) \\
& =\frac{1}{2 \pi} \int_{C}\left[G_{\varepsilon}(z, \eta) \frac{\partial G_{e}(z, \zeta)}{\partial n}-G_{e}(z, \zeta) \frac{\partial G_{\varepsilon}(z, \eta)}{\partial n}\right] d s .
\end{aligned}
$$

Using the boundary relations of $G_{\varepsilon}$ and $G_{e}$ on $C$ and Green's identity with respect to $\tilde{D}$, we find 


$$
\frac{G_{\varepsilon}(\zeta, \eta)-G_{e}(\zeta, \eta)}{\varepsilon-e}=\frac{1}{\varepsilon} G_{\varepsilon}(\zeta, \eta) \delta(\zeta)+\frac{1}{2 \pi \varepsilon} \int_{C} G_{\varepsilon}(z, \eta) \frac{\partial G_{e}(z, \zeta)}{\partial \tilde{n}} d s .
$$

Passing to the limit $\varepsilon=e$, we then obtain

$$
{ }_{\partial \varepsilon}^{\partial} G_{\varepsilon}(\zeta, \eta)=\frac{1}{\varepsilon} G_{\varepsilon}(\zeta, \eta) \delta(\zeta)+\frac{1}{2 \pi \varepsilon} \int_{C} G_{\varepsilon}(z, \eta) \frac{\partial G_{\varepsilon}(z, \zeta)}{\partial \tilde{n}} d s .
$$

The symmetry of this expression is more clearly exhibited in the form

$$
\frac{\partial}{\partial \varepsilon} G_{\varepsilon}(\zeta, \eta)=\frac{1}{2 \pi \varepsilon^{2}} \iint_{D} \nabla G_{\varepsilon}(z, \zeta) \cdot \nabla G_{\varepsilon}(z, \eta) d \tau .
$$

This result could also have been obtained by straightforward calculation from (2.19) and its analogues.

It is obvious how numerous monotonicity results can be derived from expression (22) by considering combinations with positive derivative. This formula can also be used in order to develop $G_{\varepsilon}$ in powers of $\varepsilon$. The formula is particularly useful in a more detailed discussion of the mapping functions $f_{\S}(z)$, defined in $\S 2$; however, we do not enter into this subject in the present paper.

5. An extremum problem for the Fredholm eigen values. We shall now proceed to apply the variational formulas of $\S 3$ to an important extremum problem for the lowest Fredholm eigen value of a given curve $C$. In order to explain the formulation of the problem considered we start with the following observation. Let $C$ be a three times continuously differentiable curve as was supposed throughout; if $\lambda_{1}$ is its lowest eigen value we have shown that $\lambda_{1}>1$. Now let $C^{*}$ be a continuum which consists of all points of $C$ plus a segment which has one endpoint on $C$ and the other in $D$; let $\lambda_{1}^{*}$ be its lowest eigen value. It can be shown that $\lambda_{1}^{*}=1$ however small the additional segment of $C^{*}$ is; thus two curves in an arbitrary Fréchet neighborhood can have very different lowest Fredholm eigen values.

The fact that $\lambda_{1}$ depends in this discontinuous way on its defining curve $C$ makes it difficult to frame significant extremum problems for it. The side condition on $C$ of three continuous derivatives is, on the one hand, somewhat unnatural and, on the other hand, hard to preserve under variation. We shall restrict ourselves, therefore, in this section to the consideration of analytic curves, but even in this case $\lambda_{1}$ can come as near as we wish to 1 . In fact, formula (1.45) shows that we can find ellipses with $\lambda_{1}$ arbitrarily near 1 . We have, therefore, to sharpen the concept of an analytic curve by introducing the concept of uniform analyticity of a curve. A curve $C$ is called analytic if it is mapped by a regular univalent function $z=f(\zeta)$ from the unit circum- 
ference $|\zeta|=1$. $f(\zeta)$ must be regular and univalent in some circular ring $r<|\zeta|<R$ with $r<1<R$. The class of all curves $C$ which are analytic and belong to functions $f(\zeta)$ which are regular and univalent in a fixed ring $(r, R)$ shall be called the class of uniformly analytic curves with the modulus of analyticity $(r, R)$.

Because of the normality of the family of univalent functions in a fixed region the concept of uniform analyticity lends itself easily to the construction of significant extremum problems. In particular, let us ask for the minimum value of $\lambda_{1}$ within the family of all uniformly analytic curves with modulus $(r, R)$.

We may consider our problem as an extremum problem on univalent functions. Given the class of all functions $f(\zeta)$ which are regular and univalent in $r<|\zeta|<R$, to find one in the class which maps the unit circumference onto a curve $C$ with minimum $\lambda_{1}$. The existence of such a function follows easily from the usual normality arguments and we proceed at once to characterize the extremum function by varying it and comparing it with nearby competing functions.

Since the curve $C$ mapped by the extremum function is analytic and since its $\lambda_{1}$ is obviously finite, the lowest eigen value can have only a degeneracy of finite order. Let $w_{1}^{(1)}(z), \cdots, w_{1}^{(n)}(z)$ be a complete and linearly independent set of eigen functions belonging to $\lambda_{1}$ in $D$, while $\tilde{w}_{1}^{(1)}(z), \cdots, \tilde{w}_{1}^{(n)}(z)$ are the corresponding eigen functions in $\tilde{D}$. Suppose that the image of $|\zeta|=r$ forms a continuum $\Gamma$ in $D$ while the image of $|\zeta|=R$ forms the continuum $\tilde{\Gamma}$ in $\tilde{D}$. Let $z_{0} \in \tilde{\Gamma}$; there exists an infinity of analytic functions which are univalent outside of the continuum $\tilde{\Gamma}$ and which have a series development [11]

$$
z^{*}=z+\sum_{\nu=1}^{\infty} \frac{a_{\nu} \rho^{\nu+1}}{\left(z-z_{0}\right)^{\nu}}
$$

which converges for $\left|z-z_{0}\right|>\rho$. The coefficients $a_{\nu}$ of this development are uniformly bounded

$$
\left|a_{\nu}\right| \leqq 4^{\nu+1}
$$

and $\rho$ is a positive parameter which can be chosen arbitrarily small.

Let us insert the extremum function $z=f(\zeta)$ into (1); we will thus obtain an infinity of competing functions regular and univalent in $r<|\zeta|<R$ of the form

$$
f^{*}(\zeta)=f(\zeta)+\frac{a_{1} \rho^{2}}{f(\zeta)-z_{0}}+o\left(\rho^{2}\right)
$$

They define curves $C^{*}$, the images of $|\zeta|=1$ by $f^{*}(\zeta)$. If $\lambda_{1}^{*}$ denotes the lowest eigen value of $C^{*}$, it defines a root of the secular equation 
derived in $(3.20)$ :

$$
\operatorname{det}\left\|\delta \lambda_{1} \cdot \delta_{j k}+\pi\left(\lambda_{1}^{2}-1\right) \Re\left\{a_{1} \rho^{2} \tilde{w}_{1}^{(j)}\left(z_{0}\right) \tilde{w}_{1}^{(k)}\left(z_{0}\right)\right\}\right\|=0
$$

with $\delta \lambda_{1}=\lambda_{1}^{*}-\lambda_{1}+o\left(\rho^{2}\right) . \quad \delta \lambda_{1}$ is the lowest root of $(4)$; on the other hand, we conclude from the minimum property of $C$ that

$$
\delta \lambda_{1} \geq o\left(\rho^{2}\right)
$$

and this holds, a fortiori, for all other roots of (4). Hence we can assert that the quadratic form

$$
Q_{\rho}(t)=\sum_{j, k=1}^{n} \Re\left\{a_{1} \rho^{2} \tilde{w}_{1}^{(j)}\left(z_{0}\right) \tilde{w}_{1}^{(k)}\left(z_{0}\right)\right\} t_{j} t_{k}
$$

satisfies the inequality

$$
Q_{\rho}(t) \leqq o\left(\rho^{2}\right)
$$

for every choice of the unit vector $t_{1}, \cdots, t_{n}$. Dividing by $\rho^{2}$ and passing to the limit $\rho=0$, we obtain

$$
\Re\left\{a_{1} \sum_{j, k=1}^{n} \widetilde{w}_{1}^{(j)}\left(z_{0}\right) \tilde{r}_{1}^{\prime \cdots}\left(\kappa_{j}\right) t_{j} t_{k}\right\} \leqq 0 .
$$

In particular, we obtain

$$
\Re\left\{a_{1} \tilde{w}\left(z_{0}\right)^{2}\right\} \leqq 0, \quad \tilde{w}\left(z_{0}\right)=\tilde{w}_{1}^{(1)}\left(z_{0}\right) .
$$

This inequality holds for every choice of the univalent variation function (1). We now apply the following theorem [11, 14]:

If for every point $z_{0} \in \tilde{\Gamma}$ and every univalent function (1) holds

$$
\Re\left\{a_{1} s\left(z_{0}\right)\right\} \leqq 0
$$

where $s\left(z_{0}\right)$ is regular analytic on $\tilde{\Gamma}$, then $\tilde{I}$ itself is an analytic curve $z(t)$ which satisfies the differential equation

$$
\left(\frac{d z}{d t}\right)^{2} s[z(t)]=1
$$

Hence we can deduce from $\left(8^{\prime}\right)$ that $\tilde{\Gamma}$ satisfies the differential equation

$$
\left(\frac{d z}{d t}\right)^{2} \tilde{w}[z(t)]^{2}=1
$$

In exactly the same way we prove that the extremum function $f(\zeta)$ maps the circumference $|\zeta|=r$ onto an analytic arc $\Gamma$ which satisfies the differential equation 


$$
\left(\begin{array}{l}
d z \\
d t
\end{array}\right)^{2} w[z(t)]^{2}=1
$$

Let us put

$$
z(\phi)=f\left(r e^{i \phi}\right)
$$

if $\phi$ runs from 0 to $2 \pi$ the image point $z(\phi)$ will vary over $\Gamma$. We deduce from (12) the inequality

$$
\zeta^{2} f^{\prime}(\zeta)^{2} w[f(\zeta)]^{2}<0 \quad \text { for }|\zeta|=r .
$$

Similarly, we derive from (11) the inequality

$$
\zeta^{2} f^{\prime}(\zeta)^{2} \tilde{w}[f(\zeta)]^{2}<0 \quad \text { for }|\zeta|=R .
$$

We introduce the analytic functions

$$
A(\zeta)=\zeta f^{\prime}(\zeta) w[f(\zeta)] ; \quad B(\zeta)=\zeta f^{\prime}(\zeta) \tilde{w}[f(\zeta)] .
$$

Clearly, $A(\zeta)$ is regular analytic in the ring domain $r<|\zeta|<1$ while $B(\zeta)$ is regular analytic for $1<|\zeta|<R$. (14) and (15) can be expressed as

$$
\begin{aligned}
& A(\zeta)=\text { imaginary for }|\zeta|=r \\
& B(\zeta)=\text { imaginary for }|\zeta|=R
\end{aligned}
$$

while equation (1.34) leads to

$$
-i A(\zeta)=\frac{1}{\sqrt{\lambda_{1}^{2}-1}}\left[B(\zeta)+\lambda_{1} \overline{B(\zeta)}\right] \quad \text { for }|\zeta|=1 .
$$

We have by the Schwarz' reflection principle in view of $\left(14^{\prime}\right)$ and $\left(15^{\prime}\right)$ :

$$
\overline{A(\zeta)}=-A\left(\frac{r^{2}}{\bar{\zeta}}\right), \quad \overline{B(\zeta)}=-B\left(\frac{R^{2}}{\zeta}\right)
$$

Now we can rewrite (17) into the form

$$
-i A(\zeta)=\left(\lambda_{1}^{2}-1\right)^{-1 / 2}\left[B(\zeta)-\lambda_{1} B\left(R^{2} \zeta\right)\right] \quad \text { for }|\zeta|=1,
$$

since $\bar{\zeta}=\zeta^{-1}$ for $|\zeta|=1$. By (18) we see that $A(\zeta)$ is analytic in the ring $r^{2}<|\zeta|<1$ while $B(\zeta)$ is analytic for $1<|\zeta|<R^{2}$. From (19) we can continue $B(\zeta)$ into the ring $k<|\zeta|<1$ where $k=\max \left(r^{2}, R^{-2}\right)$. By (18) again $B(\zeta)$ is, therefore, analytic in the ring $k<|\zeta|<\frac{R^{2}}{k}$ and by (19) we may continue $A(\zeta)$ beyond the unit circumference. Thus $A(\zeta)$ and $B(\zeta)$ are certainly analytic for $|\zeta|=1$. The interrelation between $A(\zeta)$ and $B(\zeta)$ is, however, best understood by the use of Laurent series 
development.

We put

$$
A(\zeta)=i \sum_{n=-\infty}^{\infty} a_{n} \zeta^{n}, \quad B(\zeta)=i \sum_{n=-\infty}^{\infty} b_{n} \zeta^{n}
$$

and are sure that both series have a ring of common convergence which contains the unit circumference. The functional equations (18) are reflected in the coefficient relations

$$
a_{-n}=\bar{a}_{n} r^{2 n}, b_{-n}=\bar{b}_{n} R^{2 n} .
$$

On the other hand, a comparison of coefficients in (19) yields

$$
-i a_{n}=\left(\lambda_{1}^{2}-1\right)^{-1 / 2}\left(1-\lambda_{1} R^{2 n}\right) b_{n} .
$$

If we replace $n$ by $-n$ and apply (21), we also find

$$
i a_{n}=\left(\lambda_{1}^{2}-1\right)^{-1 / 2}\left(R^{2 n}-\lambda_{1}\right) r^{-2 n} b_{n} .
$$

But (22) and (23) lead obviously to the alternative

$$
a_{n}=b_{n}=0 \quad \text { or } \quad \lambda_{1}=\frac{r^{2 n}+R^{2 n}}{1+(r R)^{2 n}} .
$$

Thus $A(\zeta)$ and $B(\zeta)$ are necessarily rational functions and the possible values of $\lambda_{1}$ are restricted to the various values in (24) for integer $n$. Observe that $n=0$ is excluded since $\lambda_{1}$ is surely greater than one. It is sufficient to consider only positive values of $n$ since $-n$ yields the same $\lambda_{1}$-value as $+n$. We may put equation (24) into the form

$$
\frac{\lambda_{1}-1}{\lambda_{1}+1}=\frac{R^{2 n}-1}{R^{2 n}+1} \frac{1-r^{2 n}}{1+r^{2 n}}
$$

This form makes it evident that the minimum value of $\lambda_{1}$ for fixed $r$ and $R$ is attained for $n=1$. Hence, for the lowest eigen value $\lambda_{1}$ which belongs to a uniformly analytic curve $C$ with the modulus $(r, R)$, we have established the inequality:

$$
\lambda_{1} \geq \frac{r^{2}+R^{2}}{1+(r R)^{2}} .
$$

In order to conclude the investigation we have to show that there exists, in fact, a curve $C$ within the class considered for which equality is attained in (26). This curve can be found by a careful analysis of the variational conditions (11) and (12). At first we shall state the nature of an extremum curve $C$ and compute its $\lambda_{1}$-value from its definition. Later we shall show that $C$ is uniquely determined up to linear transformations, 
Let us consider the $z$-plane slit along the linear segment $-i \mu,+i \mu$ of the imaginary axis and along the segments $|x|>1$ of the real axis. Every circular ring $r \leqq|\zeta| \leqq R$ can be mapped on such a canonical domain; the real parameter $\mu$ depends on the ratio $\frac{R}{r}$. For reasons of symmetry we can obtain that the points $\zeta=R$ and $\zeta=-R$ are mapped into $z=1$ and $z=-1$, respectively, while the points $\zeta=i r$ and $\zeta=-i r$ go into $i \mu$ and $-i \mu$. The mapping function $f(\zeta)$ has the symmetry properties :

$$
\overline{f(\zeta)}=f(\bar{\zeta}), \quad-\overline{f(\zeta)}=f(-\bar{\zeta}),
$$

and is uniquely defined. Let $C$ be the image of the unit circumference $|\zeta|=1$ under the mapping $z=f(\zeta)$. We want to prove that $C$ is the required extremum curve.

We denote again the interior and exterior of $C$ by $D$ and $\tilde{D}$, respectively. Observe that the functions

$$
W_{n}(z)=A_{n}\left(\zeta^{n}+\frac{\left(-r^{2}\right)^{n}}{\zeta^{n}}\right)
$$

are regular analytic in the entire domain $D$ while the functions

$$
\widetilde{W}_{n}(z)=B_{n}\left(\zeta^{n}+\frac{R^{2 n}}{\zeta^{n}}\right)
$$

are regular analytic in $D$. Let us define the eigen functions of $D$ and $\tilde{D}$ by

$$
w_{n}(z)=\frac{d}{d z} W_{n}(z), \quad \tilde{w}_{n}(z)=\frac{d}{d z} \widetilde{W}_{n}(z)
$$

Differentiating (28) and (29) with respect to $\zeta$, we find

$$
w_{n}[f(\zeta)] f^{\prime}(\zeta) \zeta=n A_{n}\left(\zeta^{n}-\frac{\left(-r^{2}\right)^{n}}{\zeta^{n}}\right)
$$

and

$$
\tilde{w}_{n}[f(\zeta)] f^{\prime}(\zeta) \zeta=n B_{n}\left(\zeta^{n}-\frac{R^{2 n}}{\zeta^{n}}\right)
$$

The boundary conditions (1.34) for the eigen functions of $D$ and $\tilde{D}$ will lead to the requirement

$$
-i A_{n}\left(\zeta^{n}-\frac{\left(-r^{2}\right)^{n}}{\zeta^{n}}\right)=\left(\lambda_{n}^{2}-1\right)^{-1 / 2}\left[B_{n}\left(\zeta^{n}-\frac{R^{2 n}}{\zeta^{n}}\right)+\lambda_{n} \bar{B}_{n}\left(\frac{1}{\zeta^{n}}-R^{2 n} \zeta^{n}\right)\right]
$$


for $|\zeta|=1$. This can indeed be fulfilled by satisfying the conditions

$$
\begin{aligned}
& -i A_{n}\left(\lambda_{n}^{2}-1\right)^{1 / 2}=B_{n}-\lambda_{n} R^{2 n} \bar{B}_{n} \\
& -i A_{n}\left(-r^{2}\right)^{n}\left(\lambda_{n}^{2}-1\right)^{1 / 2}=B_{n} R^{2 n}-\lambda_{n} \bar{B}_{n}
\end{aligned}
$$

which is always possible if and only if

$$
\lambda_{n}=\frac{R^{2 n}-\left(-r^{2}\right)^{n}}{\left|1-\left(-r^{2} R^{2}\right)^{n}\right|}
$$

Conversely, it is evident that the values $\lambda_{n}$ determined by (35) for $n=1,2, \cdots$ lead to actual eigen functions for the domains $D$ and $\tilde{D}$. Observe, in particular, that

$$
\lambda_{1}=\frac{R^{2}+r^{2}}{1+r^{2} R^{2}}
$$

which verifies that $C$ is indeed an extremum curve and that our estimate (26) is the best possible one.

There remains finally the uniqueness question relative to the extremum curve $C$. In order to answer it we return to the functions $A(\zeta)$ and $B(\zeta)$ connected with the extremum function $f(\zeta)$. Since we know now that in their Laurent development all coefficients vanish except for $a_{1}, a_{-1}$ and $b_{1}, b_{-1}$, we have by (16), (21) and (22)

$$
\zeta f^{\prime}(\zeta) w[f(\zeta)]=i a_{1}\left(\zeta+\frac{r^{2}}{\zeta}\right)
$$

and

$$
\zeta f^{\prime}(\zeta) \tilde{w}[f(\zeta)]=i b_{1}\left(\zeta-\frac{R^{2}}{\zeta}\right)
$$

with

$$
i a_{1}\left(\lambda_{1}^{2}-1\right)^{1 / 2}=\left(\lambda_{1} R^{2}-1\right) b_{1} .
$$

We made the unessential assumption that $a_{1}$ is real which leads to the consequence that $b_{1}$ is pure imaginary.

We integrate $\left(36^{\prime}\right)$ and $(37)$ and find

$$
W[f(\zeta)]=i a_{1}\left(\zeta-\frac{r^{2}}{\zeta}\right), \quad \widetilde{W}[f(\zeta)]=i b_{1}\left(\zeta+\frac{R^{2}}{\zeta}\right)
$$

where $W(z)$ and $\tilde{W}(z)$ are properly chosen integrals of $w(z)$ and $\tilde{w}(z)$. The function $W(z)$ is single-valued in $D ; f(\zeta)$ is regular analytic on $|\zeta|=r$ and can be continued somewhat beyond this circumference. It 
will take values near the continuum $\Gamma$ after this continuation; but these values in the $z$-plane were already attained for some values $\zeta$ in $|\zeta|>r$. Hence $W[f(\zeta)]$ must take the same values for $|\zeta|$ somewhat larger than $r$ and for some $|\zeta|$ less than $r$. From (39) we recognize that these corresponding $\zeta$-values must be connected by the equation

$$
\zeta_{1}-\frac{r^{2}}{\zeta_{1}}=\zeta_{2}-\frac{r^{2}}{\zeta_{2}}
$$

Hence we proved the functional equation for $f(\zeta)$ :

$$
f(\zeta)=f\left(-\frac{r^{2}}{\zeta}\right)
$$

In exactly the same manner we derive from the second formula (39) the functional equation

$$
f(\zeta)=f\left(\frac{R^{2}}{\zeta}\right)
$$

We know already that the extremum function $f(\zeta)$ will remain an extremum function after a linear transformation since we showed at the end of $\S 1$ that $\lambda_{1}$ does not change under linear transformations. Hence we may assume without loss of generality that

$$
f(r)=0, \quad f(R)=1, \quad f(i R)=\infty .
$$

From (41) and (42) conclude then that

$$
f(-r)=0, \quad f(-i R)=\infty
$$

and in view of the univalent character of $f(\zeta)$ in $r<|\zeta|<R$ we conclude that $f(\zeta)$ has simple zeros and simple poles at these points. It is now easy to obtain for $f(\zeta)$ a product representation in terms of its known zeros and poles in the entire $\zeta$-plane and to identify it with the function which maps the ring $r<|\zeta|<R$ on the above described slit domain. This completes the uniqueness argument.

Let us return to the inequality (26). An important special case deals with all uniformly analytic curves with the modulus $(r, \infty)$. This is the class of curves which are images of $|\zeta|=1$ mapped by functions which are regular and univalent for $|\zeta|>r$. We find the estimate

$$
\lambda_{1} \geqq r^{-2}
$$

and the extremum curve in this case is the ellipse $C$ which is obtained from $|\zeta|=1$ by the mapping

$$
z=\zeta+\frac{r^{2}}{\zeta}
$$


This follows directly from (1.45) as well as from our preceding characterization of the extremum domain. The inequality (45) can also be easily derived from the estimate $\left(3.29^{\vee}\right)$; thus this particular result could have been proved by means of a Hadamard type variational formula.

As for the class of uniformly analytic functions with the modulus $(0, R)$, we have analogously the estimate

$$
\lambda_{1} \geqq R^{2} \text {. }
$$

The extremal curve $C$ is obtained from the unit circumference by the mapping

$$
z=\frac{2 R \zeta}{R^{2}+\zeta^{2}}
$$

This mapping is best understood if we consider the intermediate step

$$
\eta=R^{-2} \zeta+\frac{1}{\zeta}
$$

which maps the unit circumference onto an ellipse with $\lambda_{1}=R^{2}$ and the circumference $|\zeta|=R$ onto the linear segment $\left\langle\begin{array}{c}2 \\ R, \\ R\end{array}\right\rangle$. The additional linear transformation $z=\frac{2}{R \eta}$ does not affect the eigen values and leads to a regular univalent function in $|\zeta|<R$. We could have obtained the mapping (48) also as a special case of the preceding characterization of the extremum curve $C$.

6. Concluding remarks. We have restricted ourselves in the present paper to the case of simply connected domains. It is possible to extend a considerable amount of the results to the case of multiplyconnected domains $[3,10,14]$. The investigation becomes, however, more complicated for two reasons. First, we will have a larger number of complementary domains and, second, we will have additional eigen functions belonging to the eigen value one. In fact, let $C_{1}, C_{2}, \cdots, C_{n}$ denote the $n$ components of the boundary $C$ of the domain $D ; \operatorname{let} \omega_{\nu}(z)$ be that harmonic function in $D$ which takes on $C_{\mu}$ the boundary value $\delta_{\nu \mu}$. Then it is easily seen that

$$
w_{\nu}(z)=i \frac{\partial}{\partial z} \omega_{\nu}(z)
$$

will satisfy the integral equation

$$
w_{\nu}(z)=\frac{1}{\pi} \iint_{\nu} \frac{\overline{w_{\nu}(z)}}{(\zeta-z)^{2}} d \tau
$$


All other eigen functions of the integral equation (1.13) belong, however, to eigen values which are larger than one.

The concept of the dielectric Green's function carries over to the case of higher multiplicity and analogous series developments in terms of the eigen functions of the Fredholm integral equation are possible. Likewise, the different variational formulas can be extended to multiple connectivity. But, clearly, it will be much more difficult to draw simple conclusions from these formulas. One has only to consider the great use made in the preceding section of Laurent series developments in order to appreciate the great simplification introduced by the assumption of a simply connected domain.

\section{REFERENCES}

1. L. V. Ahlfors, Remarls on the Neumann-Poincaré integral equation, Pacific J. Math., 2 (1952), 271-280.

2. ㄴ. Conformality with respect to Riemann metrics, Ann. Acad. Sci. Fenn., Series A 202 (1955).

3. S. Bergman and M. Schiffer, Kernel functions and conformal mapping, Compositio Math., 8 (1951), 205-249.

4. G. Birkhoff, D. M. Young and E. H. Zarantanello, Effective conformal transformation of smooth simply-connected domains, Proc. Nat. Acad. Sci., 37 (1951), 411-414.

5. - Numerical methods in conformal maspaing, Proc. Symposia in Appl. Math., IV (1953), 117-140.

6. G. F. Carrier, On a conformal mapping technique, Quart. Appl. Math., 5 (1947), 101104.

7. S. Gershgorin, On conformal mapping of a simply-connected region onto a circle, Math. Sb. 40 (1933), 48-58.

8. K. Löwner, Untersuchungen über schlichte konforme Abbildungen des Einheitskreises, Math. Ann., 89 (1923), 103-121.

9. F. Rellich, Störungstheorie der Spektralzerlegwng, 1. Mitteilung, Math. Ann., 113 (1937), 600-619.

10. H. L. Royden, A modification of the Neumann-Poincare method for multiply-connected regions, Pacific J. Math., 2 (1952), 385-394.

11. M. Schiffer, A method of variation within the family of simple functions, Proc. London Math. Soc., 44 (1938), 432-449.

12. - Variation of the Green's function and theory of the p-valued functions, Amer. J. Math., 65 (1943), 341-360.

13. Hadamard's formula and variation of domain functions, Amer. J. Math., 68 (1946), 417-448.

13. M. Schiffer and D. C. Spencer, Functionals of finite Riemann surfaces, Princeton (1954).

14. S. E. Warschawski, On the effective determination of conformal maps, Contribution to the Theory of Riemann Surfaces, Princeton (1953). 



\section{PACIFIC JOURNAL OF MATHEMATICS}

\section{EDITORS}

H. L. ROYDEN

Stanford University

Stanford, California

R. A. Beaumont

University of Washington

Seattle 5, Washington

\section{A. L. Whiteman}

University of Southern California

Los Angeles 7, California

E. G. Straus

University of California

Los Angeles 24, California

\section{ASSOCIATE EDITORS}
E. F. BECKENBACH
C. E. BURGESS
M. HALL
E. HEWITT

\author{
A. HORN \\ V. GANAPATHY IYER \\ R. D. JAMES \\ M. S. KNEBELMAN
}

L. NACHBIN

I. NIVEN

G. SZEKERES

T. G. OSTROM

M. M. SCHIFFER
F. WOLF

K. YOSIDA

\section{SUPPORTING INSTITUTIONS}

\author{
UNIVERSITY OF BRITISH COLUMBIA \\ CALIFORNIA INSTITUTE OF TECHNOLOGY \\ UNIVERSITY OF CALIFORNIA \\ MONTANA STATE UNIVERSITY \\ UNIVERSITY OF NEVADA \\ OREGON STATE COLLEGE \\ UNIVERSITY OF OREGON \\ UNIVERSITY OF SOUTHERN CALIFORNIA
}

\author{
STANFORD UNIVERSITY \\ UNIVERSITY OF UTAH \\ WASHINGTON STATE COLLEGE \\ UNIVERSITY OF WASHINGTON \\ AMERICAN MATHEMATICAL SOCIETY \\ CALIFORNIA RESEARCH CORPORATION \\ HUGHES AIRCRAFT COMPANY \\ THE RAMO-WOOLDRIDGE CORPORATION
}

Mathematical papers intended for publication in the Pacific Journal of Mathematics should be typewritten (double spaced), and the author should keep a complete copy. Manuscripts may be sent to any of the editors. All other communications to the editors should be addressed to the managing editor, E. G. Straus at the University of California, Los Angeles 24, California.

50 reprints per author of each article are furnished free of charge; additional copies may be obtained at cost in multiples of 50 .

The Pacific Journal, of Mathematics is published quarterly, in March, June, September, and December. The price per volume (4 numbers) is $\$ 12.00$; single issues, $\$ 3.50$. Back numbers are available. Special price to individual faculty members of supporting institutions and to individual members of the American Mathematical Society: $\$ 4.00$ per volume; single issues, $\$ 1.25$.

Subscriptions, orders for back numbers, and changes of address should be sent to Pacific Journal of Mathematics, 2120 Oxford Street, Berkeley 4, California.

Printed at Kokusai Bunken Insatsusha (International Academic Printing Co., Ltd.), No. 10, 1-chome, Fujimi-cho, Chiyoda-ku, Tokyo, Japan.

PUBLISHED BY PACIFIC JOURNAL OF MATHEMATICS, A NON-PROFIT CORPORATION

The Supporting Institutions listed above contribute to the cost of publication of this Journal, but they are not owners or publishers and have no responsibility for its content or policies. 


\section{Pacific Journal of Mathematics}

\section{Vol. 7, No. 2 \\ February, 1957}

William F. Donoghue, Jr., The lattice of invariant subspaces of a completely continuous quasi-nilpotent transformation ................... 1031

Michael (Mihály) Fekete and J. L. Walsh, Asymptotic behavior of restricted extremal polynomials and of their zeros.................... 1037

Shaul Foguel, Biorthogonal systems in Banach spaces ............... 1065

David Gale, A theorem on flows in networks ................... 1073

Ioan M. James, On spaces with a multiplication .................. 1083

Richard Vincent Kadison and Isadore Manual Singer, Three test problems in operator theory .................................... 1101

Maurice Kennedy, A convergence theorem for a certain class of Markoff processes........................................ 1107

G. Kurepa, On a new reciprocity, distribution and duality law ........ 1125

Richard Kenneth Lashof, Lie algebras of locally compact groups ........ 1145

Calvin T. Long, Note on normal numbers .................... 1163

M. Mikolás, On certain sums generating the Dedekind sums and their reciprocity laws ..................................... 1167

Barrett O'Neill, Induced homology homomorphisms for set-valued maps......................................... 1179

Mary Ellen Rudin, A topological characterization of sets of real numbers........................................... 1185

M. Schiffer, The Fredholm eigen values of plane domains 1187

F. A. Valentine, A three point convexity property .........

Alexander Doniphan Wallace, The center of a compact lattice is totally

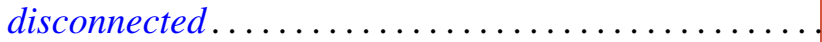

Alexander Doniphan Wallace, Two theorems on topological lattices.

G. T. Whyburn, Dimension and non-density preservation of mappings...

John Hunter Williamson, On the functional representation of certain algebraic systems ... 